\title{
SIGNATURES OF INVARIANT HERMITIAN FORMS ON IRREDUCIBLE HIGHEST WEIGHT MODULES
}

\author{
WAI LING YEE
}

\begin{abstract}
Perhaps the most important problem in representation theory in the 1970s and early 1980s was the determination of the multiplicity of composition factors in a Verma module. This problem was settled by the proof of the Kazhdan-Lusztig Conjecture which states that the multiplicities may be computed via Kazhdan-Lusztig polynomials. In this paper, we introduce signed Kazhdan-Lusztig polynomials, a variation of Kazhdan-Lusztig polynomials which encodes signature information in addition to composition factor multiplicities and Jantzen filtration level. Careful consideration of Gabber and Joseph's proof of Kazhdan and Lusztig's recursive formula for computing Kazhdan-Lusztig polynomials and an application of Jantzen's determinant formula lead to a recursive formula for the signed Kazhdan-Lusztig polynomials. We use these polynomials to compute the signature of an invariant Hermitian form on an irreducible highest weight module. Such a formula has applications to unitarity testing.
\end{abstract}

\section{INTRODUCTION}

1.1. The Unitary Dual Problem. In the 1930s, I.M. Gelfand introduced a broad programme in abstract harmonic analysis which would permit the transfer of difficult problems in areas as distinct from analysis as topology to more tractable problems in algebra. Fourier analysis is just one incarnation of this programme. An unresolved component in Gelfand's programme is the classification of the irreducible unitary representations of a group, known as the unitary dual problem.

In the case of a real reductive Lie group, the problem is equivalent to identifying all irreducible Harish-Chandra modules which admit a positive definite invariant Hermitian form. As Harish-Chandra modules may be constructed via an algebraic method introduced by Zuckerman in 1978 known as cohomological induction, it is of interest to study signatures of invariant Hermitian forms on cohomologically induced modules and to understand how positivity can fail.

Cohomological induction is a two-step process in which we compose an induction functor with a Zuckerman functor $\Gamma^{i}$. The intermediate module in cohomological induction is a generalized Verma module which admits an invariant Hermitian form if the module to which induction was applied admits an invariant Hermitian form. Formulas for the signatures of invariant Hermitian forms on these intermediate modules may be used to compute signatures of forms on corresponding cohomologically induced modules (eg. Wal84). This motivates the study of invariant Hermitian forms on Verma modules (see Wal84, Yee05) and irreducible highest weight modules.

1991 Mathematics Subject Classification. Primary 22E47.

This research was supported by an NSERC postdoctoral fellowship and by the Killam Foundation. 
1.2. Overview. Let $\mathfrak{g}_{0}$ be a real semisimple Lie algebra, $\theta$ a Cartan involution of $\mathfrak{g}_{0}, \mathfrak{g}_{0}=\mathfrak{k}_{0} \oplus \mathfrak{p}_{0}$ the corresponding Cartan decomposition, and $\mathfrak{h}_{0}=\mathfrak{t}_{0} \oplus \mathfrak{a}_{0}$ a $\theta$-stable Cartan subalgebra and corresponding Cartan decomposition (recall that every Cartan subalgebra is conjugate to one which is $\theta$-stable). We drop the subscript 0 to denote complexification. A Hermitian form $\langle\cdot, \cdot\rangle$ on a $\mathfrak{g}$-module $V$ is invariant if it satisfies

$$
\langle X v, w\rangle+\langle v, \bar{X} w\rangle=0
$$

for every $X \in \mathfrak{g}$ and every $v, w \in V$, where $\bar{X}$ denotes the complex conjugate of $X$ with respect to the real form $\mathfrak{g}_{0}$ of $\mathfrak{g}$. In this paper, we develop a formula for the signature character of an invariant Hermitian form on an irreducible highest weight module of regular infinitesimal character when it exists and $\mathfrak{h}$ is compact. In the process, we present a survey of results concerning Verma modules and the Bernstein-Gelfand-Gelfand category $\mathcal{O}$.

We describe the organization of this paper. In section 2 we review formulas for signature characters of invariant Hermitian forms on Verma modules and their relation to the Jantzen filtration. In section 3 we give a brief survey of the theory of Verma modules, define signed Kazhdan-Lusztig polynomials, and then express the signature character of an invariant Hermitian form on an irreducible highest weight module in terms of these polynomials (Theorem 3.2.3). In section 4 we develop recursive formulas for computing signed Kazhdan-Lusztig polynomials when $\mathfrak{h}$ is compact (Theorem 4.6.10). We begin by treating the elementary cases. Next, we discuss category $\mathcal{O}$, Jantzen's translation functors, Jantzen's determinant formula, and coherent continuation functors; we adapt classical results for contravariant forms to invariant Hermitian forms. Finally, we show how Gabber and Joseph's proof of the remaining "difficult" recursive formula for computing Kazhdan-Lusztig polynomials may be modified to complete a set of recursive formulas which may be used to compute signed Kazhdan-Lusztig polynomials. In section 5 we compute some examples. Throughout this paper, the results typically only require existence of non-zero invariant Hermitian forms (thus $\mathfrak{h}$ must be maximally compact in addition to $\theta$-stable). However, we only know how to compute signed Kazhdan-Lusztig polynomials when $\mathfrak{h}$ is compact because in this case, it is easy to derive formulas for various quantities from analogous formulas for contravariant forms. We impose the additional condition of compactness on $\mathfrak{h}$ in: subsection 4.1 Lemma 4.3.4 Proposition 4.3.5 and any computations of inner products from subsection 4.5 to the end of section 1

Acknowledgements. I would like to thank David Vogan for many helpful discussions, Joel Kamnitzer and Hannah Wachs for their hospitality, Alexander Postnikov for asking about irreducible highest weight modules during my thesis defence, and the referee for many helpful suggestions.

\section{Forms on Verma Modules and Filtrations}

2.1. The Signature of the Shapovalov Form on Irreducible Verma Modules. We review the contents of Yee05.

Let $\mathfrak{b}=\mathfrak{h} \oplus \mathfrak{n}$ be a Borel subalgebra of $\mathfrak{g}$. Let $\Delta^{+}(\mathfrak{g}, \mathfrak{h})$ be the set of positive roots determined by $\mathfrak{b}$ and let $\rho$ be one half the sum of the positive roots. For $\lambda \in \mathfrak{h}^{*}$, let $M(\lambda)=U(\mathfrak{g}) \otimes_{U(\mathfrak{b})} \mathbb{C}_{\lambda-\rho}$ be the Verma module of highest weight $\lambda-\rho$. We choose a generator $v_{\lambda-\rho}$ for $M(\lambda)$. Given $\mu \in \mathfrak{h}^{*}, \theta \mu$ is defined by $(\theta \mu)(H)=\mu\left(\theta^{-1} H\right)$ 
for every $H$ in $\mathfrak{h}^{*}$. The complex conjugate of $\mu, \bar{\mu}$, is defined by $\bar{\mu}(H)=\overline{\mu(\bar{H})}$. We have the relation $\theta \mu=-\bar{\mu}$ for $\mu \in \Lambda_{r}$. The Verma module $M(\lambda)$ admits an invariant Hermitian form $\langle\cdot, \cdot\rangle_{\lambda}$, which is unique up to a real scalar, when $\mathfrak{h}$ is maximally compact, $\theta\left(\Delta^{+}(\mathfrak{g}, \mathfrak{h})\right)=\Delta^{+}(\mathfrak{g}, \mathfrak{h})$ (recall that $\mathfrak{h}$ is $\theta$-stable), and $\lambda$ takes imaginary values on $\mathfrak{h}_{0}$. Normalized so that $\left\langle v_{\lambda-\rho}, v_{\lambda-\rho}\right\rangle_{\lambda}=1$, it is known as the Shapovalov form. Henceforth, we shall take $\mathfrak{h}$ and $\mathfrak{b}$ to have the properties required for existence of invariant Hermitian forms.

Define $X^{*}=-\bar{X}$ for $X \in \mathfrak{g}$, and extend ** to an anti-involution of $U(\mathfrak{g})$ via $(x y)^{*}=y^{*} x^{*}$ for $x, y \in U(\mathfrak{g})$. Then

$$
\left\langle x v_{\lambda-\rho}, y v_{\lambda-\rho}\right\rangle_{\lambda}=(\lambda-\rho)\left(p\left(y^{*} x\right)\right)
$$

where $p: U(\mathfrak{g}) \rightarrow U(\mathfrak{h})$ is defined to be projection under the direct $\operatorname{sum} U(\mathfrak{g})=$ $U(\mathfrak{h}) \oplus\left(\mathfrak{n}^{o p} U(\mathfrak{g})+U(\mathfrak{g}) \mathfrak{n}\right)$.

Due to invariance, the Shapovalov form pairs the $\lambda-\mu-\rho$ weight space of $M(\lambda)$ with the $\lambda-\theta \mu-\rho$ weight space. Recalling that $\theta \mu=-\bar{\mu}$ for $\mu \in \Lambda_{r}$, we see that these are two distinct weight spaces when $\mu$ is not imaginary, and they are the same weight space if $\mu$ is imaginary. Finite dimensionality of these spaces allows us to discuss determinants and signatures of the Shapovalov form.

A modification of the classical (invariant bilinear) Shapovalov determinant formula shows that when $\mu$ is imaginary so that the weight space $M(\lambda)_{\lambda-\mu-\rho}$ is paired with itself, the determinant of a matrix representing the Shapovalov form on $M(\lambda)_{\lambda-\mu-\rho}$ is

$$
\prod_{\alpha \in \Delta^{+}(\mathfrak{g}, \mathfrak{h})} \prod_{n=1}^{\infty}\left(\left(\lambda, \alpha^{\vee}\right)-n\right)^{P(\mu-n \alpha)}
$$

up to multiplication by a scalar determined by the basis chosen. $P$ denotes Kostant's partition function.

When $\mu$ is not imaginary (i.e. $\mu \neq \theta \mu$ ), the determinant of a matrix representing $\langle\cdot, \cdot\rangle_{\lambda}$ on $M(\lambda)_{\lambda-\mu-\rho} \oplus M(\lambda)_{\lambda-\theta \mu-\rho}$ is

$$
\prod_{\alpha \in \Delta^{+}(\mathfrak{g}, \mathfrak{h})} \prod_{n=1}^{\infty}\left(\left(\lambda, \alpha^{\vee}\right)-n\right)^{P(\mu-n \alpha)}\left(\left(\lambda, \alpha^{\vee}\right)-n\right)^{P(\theta \mu-n \alpha)}
$$

up to multiplication by a scalar.

Since $\left\langle M(\lambda)_{\lambda-\nu-\rho}, M(\lambda)_{\lambda-\nu-\rho}\right\rangle_{\lambda}=0$ when $\nu$ is not imaginary, therefore for non-imaginary $\mu$, the number of positive eigenvalues of a matrix representing the Shapovalov form on $M(\lambda)_{\lambda-\mu-\rho} \oplus M(\lambda)_{\lambda-\theta \mu-\rho}$ equals the number of negative eigenvalues of that matrix (see Sublemma 3.18 of Vog84 or p.7 of Yee05]). Thus the signature of the Shapovalov form on $M(\lambda)$ may be recorded in a formal sum called the signature character as follows:

$$
\operatorname{ch}_{s} M(\lambda)=\sum_{\substack{\mu \in \Lambda_{r}^{+} \\ \mu \text { imaginary }}}(p(\mu)-q(\mu)) e^{\lambda-\mu-\rho}
$$

where the signature of the form on $M(\lambda)_{\lambda-\mu-\rho}$ is $(p(\mu), q(\mu))$. Note that $p(\mu)+q(\mu)$ is the dimension of $M(\lambda)_{\lambda-\mu-\rho}$, whence the usual character formula when all the roots are imaginary (i.e. $\mathfrak{h}$ is compact) is

$$
\operatorname{ch} M(\lambda)=\sum_{\mu \in \Lambda_{r}^{+}}(p(\mu)+q(\mu)) e^{\lambda-\mu-\rho} .
$$


The radical of the Shapovalov form is the unique maximal submodule of $M(\lambda)$, whence Verma modules are reducible precisely when the Shapovalov form is degenerate. Thus Verma modules $M(\lambda)$ are reducible precisely on the affine hyperplanes $H_{\alpha, n}:=\left\{\lambda \mid\left(\lambda, \alpha^{\vee}\right)=n\right\}$ where $\alpha$ is a positive root and $n \in \mathbb{Z}^{+}$. Within any region defined by these reducibility hyperplanes, the signature of the Shapovalov form cannot change because the form remains non-degenerate.

The largest region for which the signature does not change is the intersection of the negative open half spaces

$$
\left(\bigcap_{\alpha \in \Pi} H_{\alpha, 1}^{-}\right) \bigcap H_{\widetilde{\alpha}, 1}^{-}
$$

with $i \mathfrak{h}_{0}^{*}$, where $H_{\alpha, n}^{-}:=\left\{\lambda \mid\left(\lambda, \alpha^{\vee}\right)<n\right\}, \widetilde{\alpha}^{\vee}$ is the highest coroot, and $\Pi$ is the set of simple roots corresponding to our choice of $\Delta^{+}$. In Wal84, Wallach used an asymptotic argument to calculate the signature character of Verma modules $M(\lambda)$ with $\lambda$ in this region, which we refer to as the Wallach region:

Theorem 2.1.1. Let imaginary $\lambda$ satisfying $\left.\lambda\right|_{\mathfrak{a}_{0}} \equiv 0$ be in the Wallach region. Then the signature character of the Shapovalov form $\langle\cdot, \cdot\rangle_{\lambda}$ on $M(\lambda)$ is

$$
\operatorname{ch}_{s} M(\lambda)=\frac{e^{\lambda-\rho}}{\prod_{\alpha \in \Delta^{+}(\mathfrak{p}, \mathfrak{t})}\left(1-e^{-\alpha}\right) \prod_{\alpha \in \Delta^{+}(\mathfrak{k}, \mathfrak{t})}\left(1+e^{-\alpha}\right)} .
$$

(In section 2 of [Yee05, we discuss compatibility with the definition of the signature character above.) The form taken by reducibility hyperplanes suggests searching for a formula for the signature character in other regions which uses the affine Weyl group. In Yee05, we defined

$$
R(\lambda):=\sum_{\mu \in \Lambda_{r}^{+}} c_{\mu} e^{\lambda-\mu}
$$

for some constants $c_{\mu}$ given by Wallach's formula so that $R(\lambda)$ is the signature character of the Shapovalov form $\langle\cdot, \cdot\rangle_{\lambda}$ when $\lambda$ lies in the Wallach region. We also defined and computed

$$
R^{A}(\lambda):=\sum_{\mu \in \Lambda_{r}^{+}} c_{\mu}^{A} e^{\lambda-\mu}
$$

so that $R^{A}(\lambda)$ is the signature character of the Shapovalov form $\langle\cdot, \cdot\rangle_{\lambda}$ when $\lambda$ belongs to the alcove $A$. We chose the fundamental Weyl chamber $\mathfrak{C}_{0}$ to be the antidominant chamber and the fundamental alcove $A_{0}$ to be in the antidominant Weyl chamber. We computed the signature of the Shapovalov form within an alcove $A$ by taking a path from $A$ to a specific alcove in the Wallach region and computing changes to the signature character for each hyperplane crossed. We refer the reader to Theorem 3.2.4 of this paper or Theorem 4.6 of Yee05 for a partial statement of the formula and to Theorem 6.12 of Yee05. for the full formula.

2.2. The Jantzen Filtration. As it is fundamental to our study of irreducible highest weight modules, we review the main tool used in computing how signatures change across a hyperplane: the Jantzen filtration. The Jantzen filtration corresponding to an analytic family of Hermitian forms $\langle\cdot, \cdot\rangle_{t}$, for $t \in(-\delta, \delta)$, on a finite 
dimensional vector space $E$ is the sequence of subspaces

$$
E=E^{\langle 0\rangle} \supset E^{\langle 1\rangle} \supset \cdots \supset E^{\langle N\rangle}=\{0\}
$$

where $e \in E^{\langle n\rangle}$ for $n \geq 0$ if there exists an analytic function $f_{e}:(-\varepsilon, \varepsilon) \rightarrow E$ for some $\varepsilon>0$ such that

(1) $f_{e}(0)=e$

(2) $\left\langle f_{e}(t), e^{\prime}\right\rangle_{t}$ vanishes to order at least $n$ at $t=0$ for any $e^{\prime} \in E$.

As weight spaces of a Verma module are finite dimensional, by taking an analytic path $\lambda_{t}:(-\delta, \delta) \rightarrow i \mathfrak{h}_{0}^{*}$ and corresponding Hermitian forms $\langle\cdot, \cdot\rangle_{\lambda_{t}}$, we may discuss Jantzen filtrations of Verma modules.

For $e, e^{\prime} \in E^{\langle n\rangle}$, define

$$
\left\langle e, e^{\prime}\right\rangle^{n}=\lim _{t \rightarrow 0} \frac{1}{t^{n}}\left\langle f_{e}(t), f_{e^{\prime}}(t)\right\rangle_{t}
$$

which is independent of choice of $f_{e}$ and $f_{e^{\prime}}$. Then

Theorem 2.2.1. (Vog84, Proposition 3.3) The form $\langle\cdot, \cdot\rangle^{n}$ on $E^{n}$ is Hermitian with radical $E^{\langle n+1\rangle}$, and therefore it induces a non-degenerate Hermitian form on $E_{\langle n\rangle}:=E^{\langle n\rangle} / E^{\langle n+1\rangle}$, which we also denote $\langle\cdot, \cdot\rangle^{n}$. Let $\left(p_{n}, q_{n}\right)$ be the signature of $\langle\cdot, \cdot\rangle^{n},(p, q)$ be the signature of $\langle\cdot, \cdot\rangle_{t}$ for $t \in(0, \delta)$, and $\left(p^{\prime}, q^{\prime}\right)$ be the signature of $\langle\cdot, \cdot\rangle_{t}$ for $t \in(-\delta, 0)$. Then

$$
\begin{aligned}
(p, q) & =\left(\sum_{n} p_{n}, \sum_{n} q_{n}\right) \quad \text { and } \\
\left(p^{\prime}, q^{\prime}\right) & =\left(\sum_{n \text { even }} p_{n}+\sum_{n \text { odd }} q_{n}, \sum_{n \text { odd }} p_{n}+\sum_{n \text { even }} q_{n}\right) .
\end{aligned}
$$

Consider an analytic path $\lambda_{t}$ such that $\lambda_{0}$ lies in exactly one reducibility hyperplane, $H_{\alpha, n}$, and $\lambda_{t}$ does not lie in any reducibility hyperplane for $t \neq 0$. Then $M\left(\lambda_{0}\right)$ has a unique proper non-trivial submodule: $M\left(\lambda_{0}-n \alpha\right)=M\left(s_{\alpha} \lambda_{0}\right)$. It lies in an odd level of the Jantzen filtration, and therefore as one crosses the hyperplane $H_{\alpha, n}$, the signature changes by the signature of an invariant Hermitian form on $M\left(\lambda_{0}-n \alpha\right)$ and thus by plus or minus the signature of $\langle\cdot, \cdot\rangle_{\lambda_{0}-n \alpha}$. We write this as

$$
R^{A}(\lambda)=R^{A^{\prime}}(\lambda)+2 \varepsilon\left(A, A^{\prime}\right) R^{A-n \alpha}(\lambda-n \alpha)
$$

for adjacent alcoves $A$ and $A^{\prime}$ separated by the hyperplane $H_{\alpha, n} . \quad \varepsilon\left(A, A^{\prime}\right)$ is a function of the Weyl chamber containing $A$ and $A^{\prime}, \alpha$, and $n$. Its value may be found in Yee05. The relation above leads to the inductive formula for the signature character of the Shapovalov form on an irreducible Verma module which was mentioned previously.

Results which we wish to use are formulated in terms of the canonical Jantzen filtration and so we review this classical concept and investigate relations to our version of the Jantzen filtration.

We use the setup of GJ81 since we will follow sections of it closely. Let $\left\{X_{\alpha}, Y_{\alpha} \mid \alpha \in \Delta^{+}(\mathfrak{g}, \mathfrak{h})\right\} \cup\left\{H_{\alpha} \mid \alpha \in \Pi\right\}$ be the Chevalley basis for $\mathfrak{g}$. We let $\mathfrak{g}_{\mathbb{Z}}$ be the integer span of the Chevalley basis. It is a Lie algebra. $\mathfrak{h}_{\mathbb{Z}}, \mathfrak{n}_{\mathbb{Z}}, \mathfrak{n}_{\mathbb{Z}}^{o p}$, and $\mathfrak{b}_{\mathbb{Z}}$ are the obvious analogues. We let $A$ be the local ring $\mathbb{C}[t]_{(t)}$. For the Lie algebra 
$a_{\mathbb{Z}}$, define $a_{A}$ to be $a_{\mathbb{Z}} \otimes_{\mathbb{Z}} A$. Jantzen defines $U\left(\mathfrak{n}^{o p}\right)_{\mathbb{Z}}$ to be the $\mathbb{Z}$-subalgebra generated by $Y_{\alpha, n}=Y_{\alpha}^{n} / n$ ! where $\alpha \in \Delta^{+}(\mathfrak{g}, \mathfrak{h})$ and $n \geq 0$. For a highest weight module $E$ with primitive generator $v$ for which $\langle v, v\rangle=1$, define $E_{\mathbb{Z}}$ to be $U\left(\mathfrak{n}^{o p}\right)_{\mathbb{Z}} v$.

Given $\lambda \in \mathfrak{h}_{A}^{*}$, we let $A_{\lambda}$ be the one dimensional $U\left(\mathfrak{h}_{A}\right)$-module on which $H \in \mathfrak{h}$ acts by multiplication by $\lambda(H)$. Extending $A_{\lambda}$ to a $U\left(\mathfrak{b}_{A}\right)$-module by allowing $X \in \mathfrak{n}_{A}$ to act by zero, we define the Verma module over $U\left(\mathfrak{g}_{A}\right)$ by $M(\lambda)_{A}:=$ $U\left(\mathfrak{g}_{A}\right) \otimes_{U\left(\mathfrak{b}_{A}\right)} A_{\lambda-\rho}$.

There is an involutive antiautomorphism $\sigma$ of $\mathfrak{g}$ so that $\sigma(H)=H$ for all $H \in \mathfrak{h}$ and such that $\sigma\left(X_{\alpha}\right)=Y_{\alpha}$ for every positive root $\alpha$. It may be extended to an antiautomorphism of $U(\mathfrak{g})$ in the same way that $\cdot{ }^{*}$ was. This leads to the canonical contravariant forms, denoted by $(\cdot, \cdot)$, on $U(\mathfrak{g})$ and $U\left(\mathfrak{g}_{A}\right)$ Verma modules with the defining properties that the forms are symmetric, bilinear, and $(x v, w)=(v, \sigma(x) w)$ for all $x$ in the universal enveloping algebra and all elements $v, w$ of the Verma module. Thus $(\cdot, \cdot)$ on $M(\lambda)$ or $M(\lambda)_{A}$ satisfies

$$
\left(x v_{\lambda-\rho}, y v_{\lambda-\rho}\right)=(\lambda-\rho)(p(\sigma(y) x)) .
$$

Compare this with (2.1.1).

Consider the $U\left(\mathfrak{g}_{A}\right)$-module $M=M(\lambda+\delta t)_{A}$ where $\lambda \in \mathfrak{h}^{*}$ and $\delta \in \mathfrak{h}^{*}$ are regular and imaginary. The Jantzen filtration of $M(\lambda+\delta t)_{A}$ is defined to be

$$
M^{(0)} \supset M^{(1)} \supset \cdots \supset M^{(N)}=\{0\}
$$

where $M^{(j)}=\left\{v \in M \mid(v, w) \in\left(t^{j}\right) \forall w \in M\right\}$. This is a filtration of $M(\lambda+\delta t)_{A}$ by $U\left(\mathfrak{g}_{A}\right)$ modules. We get a filtration on the module $\bar{M}:=M / t M$, which is isomorphic to the $U(\mathfrak{g})$ Verma module $M(\lambda)$, via $\bar{M}^{(j)}=M^{(j)} /\left(t M \cap M^{(j)}\right)$. It is the usual Jantzen filtration of $M(\lambda)$ and does not depend on the value of regular $\delta$ : in Bar83, Barbasch showed for an arbitrary non-degenerate deformation direction that the Jantzen filtration coincides with the socle filtration. We define $M_{(j)}$ and $\bar{M}_{(j)}$ as in the Hermitian case.

Lemma 2.2.2. Let $M(\lambda)^{\langle j\rangle}$ be the $j^{\text {th }}$ level of the Jantzen filtration defined by the path $\lambda_{t}=\lambda+\delta t$. Then

$$
M(\lambda)^{(j)}=M(\lambda)^{\langle j\rangle} .
$$

Proof. Note that $(\lambda+\delta t-\rho)\left(y^{*} x\right)=(\lambda+\delta t-\rho)\left(\sigma\left(\sigma\left(y^{*}\right)\right) x\right)$ so that

$$
\left\langle x v_{\lambda+\delta t-\rho}, y v_{\lambda+\delta t-\rho}\right\rangle_{\lambda+\delta t}=\left(x v_{\lambda+\delta t-\rho}, \sigma\left(y^{*}\right) v_{\lambda+\delta t-\rho}\right) .
$$

The lemma now follows from the two definitions of the Jantzen filtration and the observation that ** and $\sigma$ are bijections from $U(\mathfrak{g})$ to $U(\mathfrak{g})$.

Henceforth, bar will denote specialization at $t=0$. For the remainder of this paper, we use the classical Jantzen filtration of a Verma module and use $j$ interchangeably with $(j)$ and with $\langle j\rangle$.

\section{Verma modules and KaZhdan-Lusztig polynomials}

3.1. A Brief Overview of Verma Modules. The structure of Verma modules has been studied by a number of people (eg. Ver68, BGG71, DL77, Jan79, [BB93).

Theorem 3.1.1. ( $c f$. Dix96 Theorem 7.6.6)

$$
\operatorname{dim} \operatorname{Hom}_{\mathfrak{g}}(M(\lambda), M(\mu)) \leq 1 \text { for all } \lambda, \mu \in \mathfrak{h}^{*} .
$$


Theorem 3.1.2. (Bernstein-Gelfand-Gelfand, Dix96] Theorem 7.6.23) For $\lambda, \mu \in$ $\mathfrak{h}^{*}$,

$$
\begin{aligned}
M(\mu) \subset M(\lambda) \Longleftrightarrow \quad & \exists \alpha_{1}, \cdots, \alpha_{m} \in \Delta^{+}(\mathfrak{g}, \mathfrak{h}) \text { such that } \\
& \lambda \geq s_{\alpha_{1}} \lambda \geq \cdots \geq s_{\alpha_{m}} \cdots s_{\alpha_{1}} \lambda=\mu .
\end{aligned}
$$

(Recall that for $\mu_{1}, \mu_{2} \in \mathfrak{h}^{*}, \mu_{1} \leq \mu_{2}$ if and only if $\mu_{2}-\mu_{1} \in \Lambda_{r}^{+}$.)

Remark 3.1.3. The above conditions may not be equivalent to $\mu \in W \lambda$ and $\mu \leq \lambda$.

Verma modules have finite composition series. The composition factors of $M(\lambda)$ are $L(\mu)$ where $M(\mu) \subset M(\lambda)$ (cf. Dix96, Theorem 7.6.23). In DL77, Deodhar and Lepowsky showed that although $\operatorname{dim} \operatorname{Hom}_{\mathfrak{g}}(M(\mu), M(\lambda)) \leq 1$, it is possible for a composition factor of a Verma module to have multiplicity greater than one. In [KL79, Kazhdan and Lusztig defined polynomials $P_{x, y}$ for $x, y \in W$ known as Kazhdan-Lusztig polynomials. They famously conjectured that for $\lambda$ antidominant and regular and for $x$ and $y$ in the integral Weyl group $W_{\lambda}$ with longest element $w_{\lambda}$, the polynomials give the multiplicity of $L(y \lambda)$ as a composition factor of $M(x \lambda)$ :

$$
[M(x \lambda): L(y \lambda)]=P_{w_{\lambda} x, w_{\lambda} y}(1)
$$

from which we obtain

$$
\operatorname{ch} M(x \lambda)=\sum_{y \leq x} P_{w_{\lambda} x, w_{\lambda} y}(1) \operatorname{ch} L(y \lambda) .
$$

Furthermore, the multiplicity of $L(y \lambda)$ in the $j^{\text {th }}$ level of the Jantzen filtration of $M(x \lambda)$ is encoded by Kazhdan-Lusztig polynomials:

$$
\left[M(x \lambda)_{j}: L(y \lambda)\right]=\text { the coefficient of } q^{(\ell(x)-\ell(y)-j) / 2} \text { in } P_{w_{\lambda} x, w_{\lambda} y} .
$$

A proof of the Kazhdan-Lusztig Conjecture was perhaps the most important open problem in representation theory in the early 1980s. In Vog79b, Vogan showed that semisimplicity of $U_{\alpha} L(x \lambda)$, where $U_{\alpha} L(x \lambda)$ is defined to be the cohomology of the complex $0 \rightarrow L(x \lambda) \rightarrow \theta_{\alpha} L(x \lambda) \rightarrow L(x \lambda) \rightarrow 0$, implies the Kazhdan-Lusztig Conjecture. In GJ81, Gabber and Joseph proved that Vogan's Conjecture follows from Jantzen's Conjecture:

$$
M(x \lambda)^{j}=M\left(x s_{\alpha} \lambda\right)^{j+1} \cap M(x \lambda)
$$

for $j \geq 0, x \in W_{\lambda}, x s_{\alpha}>x$, and $\alpha \in \Pi$ such that $\left(\lambda, \alpha^{\vee}\right) \in \mathbb{Z}$. Brylinski, Kashiwara, Beilinson, and Bernstein were able to prove the Kazhdan-Lusztig Conjecture by studying the relation between Kazhdan-Lusztig polynomials and Deligne, Goresky, and MacPherson's intersection cohomology (BK81, BB81]). Beilinson and Bernstein subsequently proved Jantzen's Conjecture (BB93]) using stronger versions of these techniques.

3.2. A formula for $c_{s} L(x \lambda)$ in terms of signed Kazhdan-Lusztig polynomials. Because the radical of the Shapovalov form on $M(x \lambda)$ is $M(x \lambda)^{1}$ and $L(x \lambda)=M(x \lambda)_{0}=M(x \lambda) / M(x \lambda)^{1}$, therefore the Shapovalov form on the Verma module descends to an invariant Hermitian form, which we also call the Shapovalov form, on the irreducible highest weight module $L(x \lambda)$. Their signatures differ only by zero eigenvalues. We write $c h_{s} L(x \lambda)$ for the signature character of the Shapovalov form on $L(x \lambda)$. (We implicitly assume here that $x \lambda$ is imaginary.) 
From equation (3.1.1), one obtains the inversion formula

$$
\operatorname{ch} L(x \lambda)=\sum_{y \leq x}(-1)^{\ell(x)-\ell(y)} P_{y, x}(1) \operatorname{ch} M(y \lambda)
$$

(cf. KL79]). Because we do not know the value of $\operatorname{ch}_{s} M(y \lambda)$ when $M(y \lambda)$ is reducible, we cannot compute signature characters from the above formula. However, we may make use of our knowledge of signature characters for alcoves which contain $x \lambda$ in their closures. To illustrate this, consider the simple example of $x \lambda$ such that only the adjacent alcoves $A$ and $A^{\prime}$ contain $x \lambda$ in their closures. Let $H_{\alpha, n}$ be the reducibility hyperplane containing $x \lambda$. Recall that as one crosses the hyperplane $H_{\alpha, n}$ at $x \lambda$, the signature changes by the signature of $M(x \lambda-n \alpha)=M\left(s_{\alpha} x \lambda\right)=L\left(s_{\alpha} x \lambda\right)$. We conclude that the signature characters for the alcoves $A$ and $A^{\prime}$ evaluated at the point $x \lambda$ are $\operatorname{ch}_{s} L(x \lambda) \pm \operatorname{ch}_{s} L\left(s_{\alpha} x \lambda\right)$ in some order so that $\frac{1}{2}\left(R^{A}(x \lambda)+R^{A^{\prime}}(x \lambda)\right)=c h_{s} L(x \lambda)$. We have formulas for $R^{A}$ and for $R^{A^{\prime}}$, and so we have expressed $c h_{s} L(x \lambda)$ in terms of known quantities.

We now consider the general case. Take the path $\lambda_{t}=x \lambda+\delta t$, where $\delta$ is regular and imaginary, and consider the Jantzen filtration of $M(x \lambda)$ which it defines. Now $M(x \lambda)_{j}$ is semisimple (cf. GJ81 Theorem 4.8 (ii) ) and $\langle\cdot, \cdot\rangle_{j}$ is a non-degenerate invariant Hermitian form on $M(x \lambda)_{j}$. The contribution to the signature character of $\langle\cdot, \cdot\rangle_{j}$ by a particular irreducible constituent $L(y \lambda)$ of $M(x \lambda)_{j}$ is either the signature character of the Shapovalov form, the negative of it, or zero because $L(y \lambda)$ is paired with $L(\theta y \lambda)$ (which may be another copy of $L(y \lambda)$ ). Recording which of the three choices occurs for each composition factor with $+1,-1$, or 0 , we have

$$
c h_{s}\langle\cdot, \cdot\rangle_{j}=\sum_{y \leq x} a_{w_{\lambda} x, w_{\lambda} y, j}^{\lambda,} c h_{s} L(y \lambda)
$$

for some integers $a_{w_{\lambda} x, w_{\lambda} y, j}^{\lambda, \delta}$. Since signatures cannot change in the interior of an alcove, we will let $w(\delta) \in W_{\lambda}$ be such that $\delta \in w(\delta) \mathfrak{C}_{0}$ and we will write $a_{w_{\lambda} x, w_{\lambda} y, j}^{\lambda, w(x)}$ in place of $a_{w_{\lambda} x, w_{\lambda} y, j}^{\lambda, \delta}$ We record these integers in polynomials

$$
P_{w_{\lambda} x, w_{\lambda} y}^{\lambda, w}(q):=\sum_{j \geq 0} a_{w_{\lambda} x, w}^{\lambda, w} w_{\lambda} y, j q^{\frac{\ell(x)-\ell(y)-j}{2}}
$$

which we call the signed Kazhdan-Lusztig polynomials. We remind the reader that the signed Kazhdan-Lusztig polynomials above are indexed by a regular antidomininant weight $\lambda$ and by elements $w, x$, and $y$ of $W_{\lambda}$.

Remark 3.2.1. Note that the usual Kazhdan-Lusztig polynomials are defined in the same way, but with contributions of +1 to coefficients for every composition factor rather than $+1,-1$, or 0 . Therefore

$$
\left|a_{w_{\lambda} x, w_{\lambda} y, j}^{\lambda, w}\right| \leq\left[M(w \lambda)_{j}: L(y \lambda)\right]=\text { coefficient of } q^{(\ell(x)-\ell(y)-j) / 2} \text { in } P_{w_{\lambda} x, w_{\lambda} y} .
$$

Let $A(x \lambda, w(\delta))$ be the alcove containing $x \lambda+\delta t$ for regular, imaginary $\delta$ and for small $t>0$. Using our formulas above and Theorem 2.2.1

$$
R^{A(x \lambda, w(\delta))}(x \lambda)=\sum_{j} c h_{s}\langle\cdot, \cdot\rangle_{j}=\sum_{y \leq x} P_{w_{\lambda} x, w w_{\lambda} y}^{\lambda, w(1)}(1) h_{s} L(y \lambda) .
$$


Remark 3.2.2. When $y \lambda$ is not imaginary, neither $M(y \lambda)$ nor $L(y \lambda)$ admit non-zero invariant Hermitian forms, whence $c_{s} L(y \lambda)$ is undefined. However, in this case, each $L(y \lambda)$ is paired with some $L(\theta y \lambda)$, giving us $a_{w_{\lambda} x, w_{\lambda} y, j}^{\lambda, w(x)}=0$ and $P_{w_{\lambda} x, w_{\lambda} y}^{\lambda, w(\delta)}=0$ (see our discussion in the previous section concerning non-imaginary weights). The sum above ought to be over $y$ such that $y \lambda$ is imaginary to avoid abuse of notation.

Observe that $P_{w_{\lambda} x, w_{\lambda} x}^{\lambda, w(q)}(q)=a_{w_{\lambda} x, w_{\lambda} x, 0}^{\lambda, w(x)}=1$, and so

$$
c h_{s} L(x \lambda)=R^{A(x \lambda, w(\delta))}(x \lambda)-\sum_{\substack{y<x \\ y \lambda \text { imaginary }}} P_{w_{\lambda} x, w_{\lambda} y}^{\lambda, w(\delta)}(1) \operatorname{ch} h_{s} L(y \lambda) .
$$

Applying this formula recursively, we arrive at:

Theorem 3.2.3. If $\lambda \in \mathfrak{h}^{*}$ is regular and antidominant, then for $x \in W_{\lambda}$ such that $x \lambda$ is imaginary and for any $w \in W_{\lambda}$ :

$$
\operatorname{ch} s(x \lambda)=\sum_{\substack{y_{1}<\cdots<y_{j}=x \\ y_{k} \lambda^{\prime} s \text { imaginary }}}(-1)^{j-1}\left(\prod_{i=2}^{j} P_{w_{\lambda} y_{i}, w_{\lambda} y_{i-1}}^{\lambda, w}(1)\right) R^{A\left(y_{1} \lambda, w\right)}\left(y_{1} \lambda\right) .
$$

We recall the formula for $R^{A\left(y_{1} \lambda, w\right)}\left(y_{1} \lambda\right)$ :

Theorem 3.2.4. (Yee05, Theorems 4.6 and 6.12) Let $\Delta_{i}^{+}(\mathfrak{g}, \mathfrak{h})$ be the set of imaginary roots in $\Delta^{+}(\mathfrak{g}, \mathfrak{h})$. Subscripts or superscripts $i$ will refer to objects associated with $\Delta_{i}^{+}(\mathfrak{g}, \mathfrak{h})$. We will assume that everything (simple roots, reducibility hyperplanes, etc.) in this theorem is associated to the root system of imaginary roots. Choose the fundamental alcove $A_{0}^{i}$ of $W_{a}^{i}$ and the fundamental chamber $\mathfrak{C}_{0}^{i}$ of $W_{i}$ to contain $-\rho_{i}$. Let ${ }^{-}: W_{a}^{i} \rightarrow W_{i}$ be the homomorphism arising from the semidirect product structure $W_{a}^{i}=W_{i} \ltimes \Lambda_{i}$. Given $a \in W_{a}^{i}$, let $\widetilde{a} \in W_{i}$ be such that $a A_{0}^{i} \in \widetilde{a} \mathfrak{C}_{0}^{i}$. Let $a A_{0}^{i}=C_{0} \stackrel{r_{1}}{\rightarrow} C_{1} \stackrel{r_{2}}{\rightarrow} \ldots \stackrel{r_{\ell}}{\rightarrow} C_{\ell}=\widetilde{a} A_{0}^{i}$ be a path from $a A_{0}^{i}$ to $\widetilde{a} A_{0}^{i}$. Then for imaginary $\lambda \in A_{0}^{i}$ :

$$
\begin{aligned}
\left.\operatorname{ch}_{s} M(\lambda)\right|_{\mathfrak{a}_{0}} & =\left.\lambda\right|_{\mathfrak{a}_{0}} \text { and } \\
\left.\operatorname{ch}_{s} M(\lambda)\right|_{\mathfrak{t}_{0}} & =R^{a A_{0}^{i}}\left(\lambda \mid \mathfrak{t}_{0}\right) \\
& =\sum_{\substack{\left.S=\left\{i_{1}<\cdots i_{k}\right\} \\
\subset\{1, \ldots,\}\right\}}} \varepsilon(S) 2^{|S|} \frac{e^{\left.\overline{i_{i_{1}} r_{i_{2}} \cdots \overline{r_{i_{k}}}} r_{i_{k}} r_{i_{k-1}} \cdots r_{i_{1}} \lambda\right|_{\mathfrak{t}_{0}}-\rho}}{\prod_{\alpha \in \Delta^{+}(\mathfrak{p}, \mathfrak{t})}\left(1-e^{-\alpha}\right) \prod_{\alpha \in \Delta^{+}(\mathfrak{k}, \mathfrak{t})}\left(1+e^{-\alpha}\right)}
\end{aligned}
$$

where $\varepsilon(S)=\varepsilon\left(C_{i_{1}-1}, C_{i_{1}}\right) \varepsilon\left(\overline{r_{i_{1}}} C_{i_{2}-1}, \overline{r_{i_{1}}} C_{i_{2}}\right) \cdots \varepsilon\left(\overline{r_{i_{1}}} \cdots \overline{r_{i_{k-1}}} C_{i_{k}-1}, \overline{r_{i_{1}}} \ldots \overline{r_{i_{k-1}}} C_{i_{k}}\right)$, $\varepsilon(\emptyset)=1$, and the formula for $\varepsilon\left(C, C^{\prime}\right)$ for alcoves $C, C^{\prime}$ may be found in Theorem 6.12 of Yee05.

Since we have formulas for $R^{A}$ for any alcove $A$, therefore we can compute $c h_{s} L(x \lambda)$ as long as we can compute the integers $P_{w_{\lambda} x, w_{\lambda} y}^{\lambda, w}(1)$.

\section{Recursive Formulas for COMPUting Signed Kazhdan-Lusztig POLYNOMIALS}

4.1. Recursive formulas for the easy cases. The usual Kazhdan-Lusztig polynomials may be computed via $P_{x, x}=1, P_{x, y}=0$ when $x>y$, and by the recursive formulas:

a) $P_{w_{\lambda} x, w_{\lambda} y}=P_{w_{\lambda} x s, w_{\lambda} y}$ if $y s>y$ and $x, x s \geq y, s$ simple.

a') $P_{w_{\lambda} x, w_{\lambda} y}=P_{w_{\lambda} s x, w_{\lambda} y}$ if $s y>y$ and $x, s x \geq y, s$ simple. 
b) If $y>y s$ then

$$
\begin{gathered}
q^{c} P_{w_{\lambda} x s, w_{\lambda} y}+q^{1-c} P_{w_{\lambda} x, w_{\lambda} y}=\sum_{z \in W_{\lambda} \mid z s>z} \mu\left(w_{\lambda} z, w_{\lambda} y\right) q^{\frac{\ell(z)-\ell(y)+1}{2}} P_{w_{\lambda} x, w_{\lambda} z} \\
+P_{w_{\lambda} x, w_{\lambda} y s}
\end{gathered}
$$

where $c=1$ if $x s<x, c=0$ if $x s>x$, and $\mu\left(w_{\lambda} z, w_{\lambda} y\right)$ is the multiplicity of $L(y \lambda)$ in $M(z \lambda)_{1}$.

The initial conditions for signed Kazhdan-Lusztig polynomials are identical: $P_{x, x}^{\lambda, w}=1$ and $P_{x, y}^{\lambda, w}=0$ when $x>y$. We will find the recursive formulas to be different.

We discuss the signed Kazhdan-Lusztig polynomial analogue of case a'). We assume the Cartan subalgebra $\mathfrak{h}$ to be not only maximally compact but compact for this subsection. Thus $y \lambda=\theta(y \lambda)$ for all $y \in W_{\lambda}$. Choose $x, y \in W_{\lambda}$ and $s=s_{\alpha}$ simple so that $s y>y$ and $s x>x$. Recall that $\lambda$ is regular antidominant. We consider the Jantzen filtration corresponding to the path $\lambda_{t}=x \lambda+\delta t$ where $\delta \in w \mathfrak{C}_{0}$ and we restrict our attention to $L(y \lambda)$ in the $j+1^{\text {st }}$ level of $M(s x \lambda)$. Since $s x>x$, therefore $\left(x \lambda, \alpha^{\vee}\right)>0$, whence $M(x \lambda)$ is a submodule of $M(s x \lambda)$. The Jantzen Conjecture and our recursive formula a') tell us that all copies of $L(y \lambda)$ in the $j+1^{\text {st }}$ level of the filtration arise from the submodule $M(x \lambda)$ of $M(s x \lambda)$. Similarly, if $x s>x$ and $y s>y$ then all copies of $L(y \lambda)$ in the $j+1^{\text {st }}$ level of the Jantzen filtration of $M(x s \lambda)$ arise from the submodule $M(x \lambda)$ of $M(s x \lambda)$. We obtain:

Proposition 4.1.1. Let $s=s_{\alpha}$ be a simple reflection and let $y<x$.

a) $a_{w_{\lambda} x, w_{\lambda} y, j+1}^{\lambda, w}=\operatorname{sgn}(-w \rho, x \alpha) \varepsilon\left(H_{x \alpha,\left(x s \lambda, x \alpha^{\vee}\right)}, x s\right) a_{w_{\lambda} x, w_{\lambda} y, j}^{\lambda, w}$ if $x s>x$

a') $a_{w_{\lambda} s x, w_{\lambda} y, j+1}^{\lambda, w}=\operatorname{sgn}(-w \rho, \alpha) \varepsilon\left(H_{\alpha,\left(s x \lambda, \alpha^{\vee}\right)}, s x\right) a_{w_{\lambda} x, w_{\lambda} y, j}^{\lambda, w}$ if $s x>x$.

Proof. Recall that $\varepsilon\left(A, A^{\prime}\right)$ is a function of the Weyl chamber containing $A$ and $A^{\prime}$ and the hyperplane which separates them. We therefore defined $\varepsilon\left(H_{\gamma, N}, z\right)$ for $z \in W$ in Yee05. Take an analytic path $\lambda_{t}:(-\varepsilon, \varepsilon) \rightarrow i \mathfrak{h}_{0}^{*}$ in the Weyl chamber $z \mathfrak{C}_{0}$ so that $\lambda_{t} \in H_{\gamma, N}^{+}$for $t>0, \lambda_{t} \in H_{\gamma, N}^{-}$for $t<0, H_{\gamma, N}$ is the only reducibility hyperplane containing $\lambda_{0}$, and $M\left(\lambda_{t}\right)$ is irreducible for $t \neq 0$. Let $t_{1} \in(0, \varepsilon)$ and let $t_{2} \in(-\varepsilon, 0)$. Recall that

$$
\operatorname{ch}_{s} M\left(\lambda_{t_{1}}\right)=e^{\lambda_{t_{1}}-\lambda_{t_{2}}} \operatorname{ch} M\left(\lambda_{t_{2}}\right)+2 \varepsilon\left(H_{\gamma, N}, z\right) e^{\lambda_{t_{1}}-\lambda_{0}} \operatorname{ch}_{s} M\left(\lambda_{0}-N \gamma\right)
$$

which reflects the change of the signature character by the signature character of the radical $M\left(\lambda_{0}-N \gamma\right) \subset M\left(\lambda_{0}\right)$ as we cross the reducibility hyperplane $H_{\gamma, N}$ (cf. Yee05, Proposition 3.2). Recall that $\varepsilon\left(H_{\gamma, N}, z\right)$ encodes information about singular vectors: if $f \in U\left(\mathfrak{n}^{o p}\right)$ is such that $f v_{\lambda_{0}}$ generates $M\left(\lambda_{0}-N \gamma\right)=M\left(s_{\gamma} \lambda_{0}\right)$, then

$$
\operatorname{sgn}\left\langle f v_{\lambda_{t}-\rho}, f v_{\lambda_{t}-\rho}\right\rangle_{\lambda_{t}}= \begin{cases}\varepsilon\left(H_{\gamma, N}, z\right) & \text { if } \lambda_{t} \in H_{\gamma, N}^{+} \\ -\varepsilon\left(H_{\gamma, N}, z\right) & \text { if } \lambda_{t} \in H_{\gamma, N}^{-}\end{cases}
$$

Invariant Hermitian forms on Verma modules are unique up to a real scalar, which is determined by the inner product of a generator with itself. The proposition now follows from the observation that $x \lambda=s_{\alpha} s x \lambda$ and $x \lambda=s_{x \alpha} x s \lambda$.

Corollary 4.1.2. Letting $s=s_{\alpha}$ be a simple reflection, the signed Kazhdan-Lusztig polynomials satisfy: 
a) $P_{w_{\lambda} x, w_{\lambda} y}^{\lambda, w}=\operatorname{sgn}(-w \rho, x \alpha) \varepsilon\left(H_{x \alpha,\left(x s \lambda, x \alpha^{\vee}\right)}, x s\right) P_{w_{\lambda} x s, w_{\lambda} y}^{\lambda, w}$ if $y s>y$ and $x, x s \geq$ $y$

$\left.\mathrm{a}^{\prime}\right) P_{w_{\lambda} x, w_{\lambda} y}^{\lambda, w}=\operatorname{sgn}(-w \rho, \alpha) \varepsilon\left(H_{\alpha,\left(s x \lambda, \alpha^{\vee}\right)}, s x\right) P_{w_{\lambda} s x, w_{\lambda} y}^{\lambda, w}$ if $s y>y$ and $x, s x \geq$ $y$.

An excellent companion for the remainder of this section is GJ81 from which the results of this section are derived. The objective is to compute $P_{w_{\lambda} x, w_{\lambda} y}^{\lambda_{2}}$ for case b). We begin by introducing some background material.

4.2. Category $\mathcal{O}$. Bernstein-Gelfand-Gelfand defined category $\mathcal{O}$ in BGG76. It is the subcategory of the category of $\mathfrak{g}$-modules consisting of modules $M$ satisfying:

(1) $M=\oplus_{\mu \in \mathfrak{h} *} M_{\mu}$

(2) $M$ is finitely generated

(3) $M$ is $\mathfrak{n}$-finite (i.e. $U(\mathfrak{n}) v$ is finite-dimensional for every $v \in M$ ).

Category $\mathcal{O}$ is closed under arbitrary direct sums, quotients, submodules, and tensoring with finite-dimensional modules. Verma modules are objects in category $\mathcal{O}$ and the simple objects of $\mathcal{O}$ consist of the irreducible highest weight modules $L(\mu)$ where $\mu \in \mathfrak{h}^{*}$. Irreducible highest weight modules form an additive basis of the Grothendieck group of category $\mathcal{O}$.

$\mathfrak{h}^{*}$ is a disjoint union of $W$-orbits which are called blocks. Recall that $\chi_{\mu}=\chi_{\nu}$ if and only if $\nu \in W \mu$. For each block $D$ and some $\mu \in D$, we define

$\mathcal{O}_{D}:=\mathcal{O}_{\mu}:=\left\{\right.$ modules $\in \mathcal{O} \mid \exists N$ such that $\left(z-\chi_{\mu}\right)^{N}$ annihilates $\left.M \forall z \in Z(\mathfrak{g})\right\}$.

Remark 4.2.1. These blocks are larger than the standard ones in ring theory: two irreducible modules belong to the same block if they admit a non-trivial extension in the category. In the case of category $\mathcal{O}$, this amounts to two irreducible highest weight modules having the same infinitesimal character and all of their weights differing by sums of roots.

Category $\mathcal{O}$ decomposes into blocks $\mathcal{O}_{D}$ of category $\mathcal{O}$ :

Theorem 4.2.2. (cf. [BGG76], property 4) of Section 3)

$$
\mathcal{O}=\bigoplus_{\text {blocks } D} \mathcal{O}_{D} .
$$

Denote projection onto $\mathcal{O}_{D}\left(\right.$ or $\left.\mathcal{O}_{\mu}\right)$ by $\operatorname{Pr}_{D}$ (resp. $\left.P r_{\mu}\right)$. Projection onto the blocks of category $\mathcal{O}$ defines what is known as the primary decomposition

$$
M \simeq \bigoplus_{\text {blocks } D} \operatorname{Pr}_{D} M
$$

of a module $M$ in $\mathcal{O}$. For any $M \in \mathcal{O}, \operatorname{Pr}_{D} M$ is non-zero for finitely many $D$. $\operatorname{Pr}_{D} M$ is called the primary component of $M$ with respect to the block $D$.

Theorem 4.2.3. (cf. [Jan74, Satz 1, iv) of Section 3.) Primary decomposition of a module in category $\mathcal{O}$ which admits an invariant Hermitian form is an orthogonal decomposition into submodule pairs or singletons. Specifically,

$$
\operatorname{Pr}_{D} M \text { and } \operatorname{Pr}_{D^{\prime}} M \text { are orthogonal for } D^{\prime} \neq-\bar{D} \text {. }
$$

Proof. It is straightforward to modify Jantzen's proof that primary decomposition of modules in category $\mathcal{O}$ admitting a contravariant form is an orthogonal decomposition. 
4.3. Jantzen's translation functors and his determinant formula. For an integral weight $\mu$, let $F(\mu)$ denote the finite-dimensional representation of extremal weight $\mu$. Jantzen's translation functors are compositions of tensoring with a finitedimensional module with projections onto blocks of category $\mathcal{O}$ :

$$
\begin{aligned}
T_{\lambda}^{\lambda+\mu}:\{\text { modules of inf'l character } \lambda\} & \rightarrow \text { \{modules of inf'l character } \lambda+\mu\} \\
M & \mapsto \operatorname{Pr}_{\lambda+\mu}(M \otimes F(\mu))
\end{aligned}
$$

for any $\lambda \in \mathfrak{h}^{*}$ (cf. [Kos75]). We will later use $T_{D}^{D^{\prime}}$ in place of $T_{\lambda}^{\lambda^{\prime}}$ when $\lambda$ and $\lambda^{\prime}$ are both antidominant, and therefore the value of $\lambda^{\prime}-\lambda$ may be recovered.

Recall for $\lambda \in \mathfrak{h}^{*}$ that $\Delta_{\lambda}(\mathfrak{g}, \mathfrak{h})=\left\{\alpha \in \Delta(\mathfrak{g}, \mathfrak{h}) \mid\left\langle\lambda, \alpha^{\vee}\right\rangle \in \mathbb{Z}\right\}$. A facette $\mathcal{F}$ is a non-empty subset of some $\mathbb{A}(\lambda):=\mathbb{Q} \Delta_{\lambda}(\mathfrak{g}, \mathfrak{h}) \otimes_{\mathbb{Q}} \mathbb{R}$ associated with a disjoint union $\Delta_{\lambda}^{+}(\mathfrak{g}, \mathfrak{h})=\Delta_{\mathcal{F}}^{0} \cup \Delta_{\mathcal{F}}^{+} \cup \Delta_{\mathcal{F}}^{-}:$

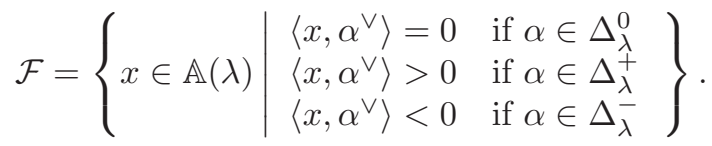

If $M$ is irreducible, $\lambda$ and $\lambda+\mu$ are antidominant with $\lambda+\mu$ in the closure of the facette containing $\lambda$, then $T_{\lambda}^{\lambda+\mu} M$ is irreducible or zero (Jan79, Theorem 2.11). If both $\lambda$ and $\lambda+\mu$ are strictly antidominant, then $T_{\lambda}^{\lambda+\mu}: \mathcal{O}_{\lambda} \rightarrow \mathcal{O}_{\lambda+\mu}$ is an equivalence of categories.

We may extend the translation functor to category $\mathcal{O}$ :

$$
\begin{aligned}
T_{\lambda}^{\lambda+\mu}: \mathcal{O} & \rightarrow \mathcal{O} \\
M & \mapsto \operatorname{Pr}_{\lambda+\mu}\left(F(\mu) \otimes\left(\operatorname{Pr}_{\lambda} M\right)\right) .
\end{aligned}
$$

In order to study how Jantzen's translation functors affect Verma modules and forms on Verma modules, we need some facts about the tensor product of a Verma module $M(\lambda)$ with a finite-dimensional module $F$ :

Theorem 4.3.1. (Bernstein-Gelfand-Gelfand, Dix96, Theorem 7.6.14.) Let $\mu_{1}$, $\ldots, \mu_{N}$ be an ordering of the weights of $F$ (with multiplicity) such that $\mu_{i} \leq \mu_{j}$ implies that $i \leq j$. Then there is a filtration of $M=M(\lambda) \otimes F$ by Verma modules:

$$
M=M^{0} \supset M^{1} \supset \cdots \supset M^{N} \supset M^{N+1}=\{0\}
$$

where $M^{i} / M^{i+1} \simeq M\left(\lambda+\mu_{i}\right)$.

Theorem 4.3.2. (Jan74, Satz 1, iii) of Section 3.) Let $M_{i}$ be $\operatorname{Pr}_{\lambda+\mu_{i}} M$. Then $M_{i}$ is generated as a $U\left(\mathfrak{n}^{o p}\right)$-module by the images of $v_{\lambda-\rho} \otimes F_{\nu}$ where $\nu$ is a weight of $F$ such that $\lambda+\nu$ belongs to the Weyl group orbit of $\lambda+\mu_{i}$.

Suppose the modules $U$ and $V$ admit an invariant Hermitian (resp. contravariant) form. The tensor product of the two modules $U \otimes V$ naturally has an invariant Hermitian (resp. contravariant) form: $\left\langle u_{1} \otimes v_{1}, u_{2} \otimes v_{2}\right\rangle_{U \otimes V}=\left\langle u_{1}, u_{2}\right\rangle_{U} \cdot\left\langle v_{1}, v_{2}\right\rangle_{V}$. Since primary decomposition of a module is orthogonal with respect to invariant Hermitian forms in the case of a compact Cartan and also with respect to contravariant forms, the $M_{i}$ s inherit invariant Hermitian forms from invariant Hermitian forms on $M(\lambda)$ and on $F$, and they inherit contravariant forms from contravariant forms on $M(\lambda)$ and on $F$. Jantzen has a determinant formula for such contravariant forms: 
Theorem 4.3.3. (Jan74, Section 5.) Suppose the numbers $\left(\lambda, \alpha^{\vee}\right)$ for $\alpha \in \Pi$ are algebraically independent over $\mathbb{Q}$. Suppose $F=L\left(\lambda_{0}\right)$ where $\lambda_{0} \in \Lambda$ is strictly dominant and let $v_{0}$ be a highest weight vector of $F$ so that $\left(v_{0}, v_{0}\right)=1$. Let $n(\mu)$ denote the multiplicity of the weight $\mu$ in $F$ and let $\left\{e_{\mu, j}\right\}_{1 \leq j \leq n(\mu)}$ be a $\mathbb{Z}$-basis of the $\mu$ weight space of $U\left(\mathfrak{n}^{o p}\right)_{\mathbb{Z}} v_{0} . \mu=\mu_{i}$ for some $i$. Denote by $f_{\mu, j}$ the orthogonal projection of $v_{\lambda-\rho} \otimes e_{\mu, j}$ onto $M_{i}$. The determinant of the contravariant form for the $f_{\mu, j}$ is $D_{F}(\mu) a_{\mu}$, where $D_{F}(\mu)$ is the determinant of the contravariant form with respect to a $\mathbb{Z}$-basis of $U\left(\mathfrak{n}^{o p}\right)_{\mathbb{Z}} v_{0}$, and thus for the $e_{\mu, j}$, and

$$
a_{\mu}=\prod_{\alpha \in \Delta^{+}(\mathfrak{g}, \mathfrak{h})} \prod_{r>0, r+\left\langle\mu, \alpha^{\vee}\right\rangle \geq 0}\left(\frac{\left(\lambda, \alpha^{\vee}\right)-r}{\left(\lambda+\mu, \alpha^{\vee}\right)+r}\right)^{n(\mu+r \alpha)} .
$$

We now compare the canonical contravariant form with the Shapovalov form for the purpose of stating Jantzen's determinant formula for invariant Hermitian forms. We begin by introducing a $\mathbb{Z}_{2}$-grading of $\Lambda_{r}$ in the case of a compact Cartan. From $[\mathfrak{k}, \mathfrak{k}] \subset \mathfrak{k},[\mathfrak{k}, \mathfrak{p}] \subset \mathfrak{p},[\mathfrak{p}, \mathfrak{p}] \subset \mathfrak{k}$, and from $\left[\mathfrak{g}_{\alpha}, \mathfrak{g}_{\beta}\right] \subset \mathfrak{g}_{\alpha+\beta}$, we see that a root is non-compact if and only if when expressed as a sum of simple roots, there are an odd number of non-compact roots in the sum, counting multiplicity. It follows that for any $\mu \in \Lambda_{r}$, the parity of the number of non-compact roots in any expression of $\mu$ as a sum of roots is independent of the expression chosen. We will denote the grading defined by this parity by $\varepsilon: \Lambda_{r} \rightarrow \mathbb{Z}_{2}$.

The Chevalley basis may be chosen so that

$$
X_{\alpha}^{*}=-\bar{X}_{\alpha}=\theta Y_{\alpha}=(-1)^{\varepsilon(\alpha)} Y_{\alpha}=(-1)^{\varepsilon(\alpha)} \sigma\left(X_{\alpha}\right)
$$

(cf. Yee05). Thus

Lemma 4.3.4. If $\mathfrak{h}$ is compact and we choose a $\mathbb{Z}$-basis from $U\left(\mathfrak{n}^{o p}\right)_{\mathbb{Z}} v_{\lambda-\rho}$ for $\left(M(\lambda)_{\mathbb{Z}}\right)_{\mu}$, matrices representing the canonical contravariant form and the invariant Hermitian form with respect to this basis differ by multiplication by the scalar $(-1)^{\varepsilon(\lambda-\rho-\mu)}$.

Proposition 4.3.5. When $\mathfrak{h}$ is compact, Theorem 4.3.3 holds with "invariant Hermitian form" in place of "contravariant form" and $(-1)^{\varepsilon\left(\lambda_{0}-\rho-\mu\right) n(\mu)} a_{\mu}$ in place of $a_{\mu}$.

Proof. A vector of weight $\lambda-\rho+\mu$ in $M(\lambda) \otimes L\left(\lambda_{0}\right)$ must be the sum of tensor products of a vector of weight $\lambda-\rho-\nu$ and a vector of weight $\mu+\nu$ for some $\nu \in \Lambda_{r}$. The proposition now follows from the grading, the lemma and the observation that $(-1)^{\varepsilon(\nu)+\varepsilon\left(\lambda_{0}-\rho-(\mu+\nu)\right)}=(-1)^{\varepsilon\left(\lambda_{0}-\rho-\mu\right)}$.

4.4. Gabber and Joseph's generalization of category $\mathcal{O}$. For the purpose of studying the Kazhdan-Lusztig Conjecture, Gabber and Joseph introduced modifications of category $\mathcal{O}$. Let $C \subset \mathfrak{h}_{A}^{*}$ be of the form $\lambda+\Lambda_{r}$ (recall the discussion of the setup of [GJ81] after Theorem 2.2.1]. Let $K_{C}$ be the subcategory of $U\left(\mathfrak{g}_{A}\right)$-modules $M$ such that:

1) $M=\sum_{\mu \in C-\rho} M_{\mu}$

2) $M$ is $U\left(\mathfrak{n}_{A}\right)$-finite

3) $M$ is finitely generated over $U\left(\mathfrak{g}_{A}\right)$.

Note that $M(\lambda)_{A}$ belongs to $\mathrm{Ob} K_{C}$. 
If $\mu \in C$, given any maximal ideal $m$ of $A$, there is a unique maximal submodule of $M(\mu)_{A}$ containing $m M(\mu)_{A}$ (cf. 1.7.2 of G.J81]). Call the corresponding simple quotient $L(m, \mu)$. In our case, $A$ is a local ring, so we use $L(\mu)_{A}$ in place of $L((t), \mu)$.

A block in the context of the category $K_{C}$ is a subset $D$ of $C$ whose specialization at $t=0, \bar{D}=\{\bar{\lambda} \mid \lambda \in D\}$, is a $W$-orbit. Define

$$
J_{D}=\bigcap_{\mu \in D} \operatorname{ker} \chi_{\mu} .
$$

We may define, as we did for category $\mathcal{O}$, the primary component of $M \in \mathrm{Ob} K_{C}$ with respect to the block $D$ :

$$
\operatorname{Pr}_{D} M=\left\{m \in M \mid \text { for all } z \in J_{D}, \text { exists } n \in \mathbb{Z}^{+} \text {such that } z^{n} m=0\right\} .
$$

We note that $C=\coprod_{i} D_{i}$ is a countable union of blocks. As for category $\mathcal{O}$ :

Proposition 4.4.1. (cf. Proposition 1.8.4, G.J81) For $M \in \mathrm{Ob} K_{C}$, we have

$$
M=\oplus_{i} \operatorname{Pr}_{D_{i}} M
$$

the primary decomposition of $M$.

Given a block $D \subset C$, the subcategory $K_{D}$ of $K_{C}$ consists of modules whose simple quotients are among the $L(m, \mu)$ where $m$ is a maximal ideal of $A$ and $\mu \in D$. $\operatorname{Pr}_{D}$ takes objects in $K_{C}$ to objects in $K_{D}$.

In GJ81, Gabber and Joseph extended Jantzen's definition of translation functors to category $K_{C}$. Let $D=W_{\lambda} \lambda+\delta$, where $\lambda, \delta \in \mathfrak{h}^{*}$ are regular and $\lambda$ is antidominant. Let $D^{\prime}=W_{\lambda}(\lambda-\mu)+\delta t$, where $\mu \in \Lambda$ and $\lambda-\mu$ is antidominant.

$$
T_{D}^{D^{\prime}} M=\operatorname{Pr}_{D^{\prime}}\left(F(-\mu)_{A} \otimes_{A}\left(\operatorname{Pr}_{D} M\right)\right)
$$

is the translation functor from the block $K_{D}$ to the block $K_{D^{\prime}}$.

We refer the reader to Definition 2.3 of Yee05 for the definition of the Hermitian dual of a module. Given a module $M$ in $\mathrm{Ob}_{\bar{C}}$, we define $\delta^{h}(M)$ to be the $\mathfrak{h}$-finite part of its Hermitian dual $M^{h}$.

Lemma 4.4.2. Let $M \in \mathrm{Ob} K_{\bar{D}}$ for some block $D \subset C$. If $M$ admits a nondegenerate invariant Hermitian form and $\mathfrak{h}$ is compact, then $\delta^{h}(M) \cong M$.

Proof. We may modify Section 3.10 and Lemma 4.7 (iii) of GJ81.

4.5. Coherent continuation functors. Suppose $\lambda \in \mathfrak{h}^{*}$ is antidominant and regular and $\delta \in \mathfrak{h}^{*}$ is regular. Let $D=W_{\lambda} \lambda+\delta t$. Let $s=s_{\alpha}$ be a simple reflection in $W_{\lambda}$. We may choose $\nu_{\alpha} \in \Lambda$ so that $\lambda-\nu_{\alpha}$ is antidominant and so that the only $\operatorname{root} \beta$ for which $\left(\lambda-\nu_{\alpha}, \beta\right)=0$ is $\beta=\alpha$. Let $D_{\alpha}=W_{\lambda}\left(\lambda-\nu_{\alpha}\right)+\delta t$. Since we are studying invariant Hermitian forms, we assume furthermore that $\delta$ and $\lambda$ are imaginary, although the statements which follow hold for non-imaginary $\delta$ and $\lambda$ if they contain no reference to invariant Hermitian forms. We fix this notation for the remainder of this article. The generalized notion (it exists for category $\mathcal{O}$ also) of translation to the $\alpha$ wall is the functor

$$
T_{D}^{D_{\alpha}} M=\operatorname{Pr}_{D_{\alpha}}\left(F\left(-\nu_{\alpha}\right)_{A} \otimes_{A}\left(\operatorname{Pr}_{D} M\right)\right)
$$

and

$$
T_{D_{\alpha}}^{D} M=\operatorname{Pr}_{D}\left(F\left(\nu_{\alpha}\right)_{A} \otimes_{A}\left(\operatorname{Pr}_{D_{\alpha}} M\right)\right)
$$

is translation from the $\alpha$ wall. Translation to the $\alpha$ wall followed by translation from the $\alpha$ wall, denoted by $\theta_{\alpha}=T_{D_{\alpha}}^{D} T_{D}^{D_{\alpha}}$, is an exact functor known as coherent 
continuation across the $\alpha$ wall or the reflection functor across the $\alpha$ wall. We will also use $\theta_{\alpha}$ to denote coherent continuation in category $\mathcal{O}$. Due to results in sections 4.2 and 4.3 if $M$ carries an invariant Hermitian form, then so do $T_{D}^{D_{\alpha}} M$, $T_{D_{\alpha}}^{D} M$, and $\theta_{\alpha} M$ naturally. We use $T\langle\cdot, \cdot\rangle$ to denote the form which results from application of the translation functor $T$ to a module with invariant Hermitian form $\langle\cdot, \cdot\rangle$.

We would like to describe the form which arises from a translation functor when the module and its invariant Hermitian form are a Verma module and its Shapovalov form respectively. For any $z \in W_{\lambda}$,

$$
T_{D}^{D_{\alpha}} M(z \lambda+\delta t)_{A} \simeq M\left(z\left(\lambda-\nu_{\alpha}\right)+\delta t\right)_{A}
$$

by Satz 2.9 of Jan79. We define

$$
\begin{gathered}
v_{z\left(\lambda-\nu_{\alpha}\right)+\delta t-\rho}^{\prime}:=\operatorname{Pr}_{D_{\alpha}} v_{z \lambda+\delta t-\rho} \otimes e_{-z \nu_{\alpha}, 1}, \\
\langle\cdot, \cdot\rangle_{z\left(\lambda-\nu_{\alpha}\right)+\delta t}^{\prime}:=T_{D}^{D_{\alpha}}\langle\cdot, \cdot\rangle_{z \lambda+\delta t}, \quad \text { and } \\
c_{z}^{\prime}:=\left\langle v_{z\left(\lambda-\nu_{\alpha}\right)+\delta t-\rho}^{\prime}, v_{z\left(\lambda-\nu_{\alpha}\right)+\delta t-\rho}^{\prime}\right\rangle_{z\left(\lambda-\nu_{\alpha}\right)+\delta t}^{\prime} .
\end{gathered}
$$

Let $\lambda_{\alpha}^{-} \in \Lambda^{+}$be the highest weight of $F\left(-\nu_{\alpha}\right)$. According to Theorems 4.3.2 and 4.3.3 and Lemma 4.3.4 the form $\langle\cdot, \cdot\rangle_{z\left(\lambda-\nu_{\alpha}\right)+\delta t}^{\prime}$ on $M\left(z\left(\lambda-\nu_{\alpha}\right)+\delta t\right)_{A}$ is such that

$$
c_{z}^{\prime}=(-1)^{\varepsilon\left(\lambda_{\alpha}^{-}+z \nu_{\alpha}\right)} D_{F\left(-\nu_{\alpha}\right)}\left(-z \nu_{\alpha}\right) a_{-z \nu_{\alpha}}^{\prime}
$$

where

$$
a_{-z \nu_{\alpha}}^{\prime}=\prod_{\beta \in \Delta^{+}(\mathfrak{g}, \mathfrak{h})} \prod_{r>0, r+\left\langle-z \nu_{\alpha}, \beta^{\vee}\right\rangle \geq 0}\left(\frac{\left(z \lambda+\delta t, \beta^{\vee}\right)-r}{\left(z \lambda-z \nu_{\alpha}+\delta t, \beta^{\vee}\right)+r}\right)^{n\left(-z \nu_{\alpha}+r \beta\right)} .
$$

On the level of signature characters, we have

$$
c h_{s}{\overline{\langle\cdot, \cdot\rangle_{z}}}_{z\left(\lambda-\nu_{\alpha}\right)+\delta t}^{\prime}=\operatorname{sgn}\left(c_{z}^{\prime}\right) c h_{s}\langle\cdot, \cdot\rangle_{z\left(\lambda-\nu_{\alpha}\right)} .
$$

Here, we observe that Jantzen's determinant formula holds in the category $K_{C}$ setting also since we work with $U\left(\mathfrak{n}^{o p}\right)_{\mathbb{Z}}$ bases and hence his projection formulas and recursive formulas hold (cf. [Jan74], Section 5).

Returning to the problem of developing a recursive formula for signed KazhdanLusztig polynomials in case b), we fix $x, y \in W_{\lambda}$ such that $y>y$ s and $x<x$ s for the remainder of this section. Following the notation of [GJ81, let $X=M(x s \lambda+\delta t)_{A}$ and let $Z=M(x \lambda+\delta t)_{A}$. Define $Y$ to be $\theta_{\alpha} Z$. Then:

Proposition 4.5.1. (GJ81, section 3.6.)

i) $\theta_{\alpha} X \simeq \theta_{\alpha} Z \simeq Y$.

ii) There is a short exact sequence $0 \rightarrow X \rightarrow Y \stackrel{\pi}{\rightarrow} Z \rightarrow 0$.

Remark 4.5.2. Because of the short exact sequence, $Y$ is called an extension of $X$ by $Z$.

Remark 4.5.3. Gabber and Joseph's results are for contravariant forms. The invariant Hermitian form analogues of their results hold in the compact Cartan case: their proofs may be transferred to the Hermitian form setting using Lemma 2.2.2 and Theorem 4.2.3 
We review Gabber and Joseph's discussion of the filtration of $Y$ by Verma modules. Now for $x^{\prime} \in W_{\lambda}$,

$$
\left[T_{D_{\alpha}}^{D} M\left(x\left(\lambda-\nu_{\alpha}\right)+\delta t\right)_{A}: M\left(x^{\prime} \lambda+\delta t\right)_{A}\right]=\operatorname{dim}\left(F\left(\nu_{\alpha}\right)_{A}\right)_{\mu}
$$

where $\mu=x^{\prime} \lambda-x\left(\lambda-\nu_{\alpha}\right)=x^{\prime} \lambda-x s\left(\lambda-\nu_{\alpha}\right)$. According to Satz 2.9 of Jan79, there are two solutions: $x^{\prime}=x, \mu=x \nu_{\alpha}$ and $x^{\prime}=x s, \mu=x s \nu_{\alpha}$. By Theorem 4.3.2 $Y$ is generated as a $U\left(\mathfrak{n}^{o p}\right)$ module by $v_{x \lambda+\delta t-\rho}^{\prime \prime}=\operatorname{Pr}_{D_{\alpha}}\left(v_{x\left(\lambda-\nu_{\alpha}\right)+\delta t-\rho}^{\prime} \otimes e_{x \nu_{\alpha}, 1}\right)$ and by $v_{x s \lambda+\delta t-\rho}^{\prime \prime}=\operatorname{Pr}_{D_{\alpha}}\left(v_{x\left(\lambda-\nu_{\alpha}\right)+\delta t-\rho}^{\prime} \otimes e_{x s \nu_{\alpha}, 1}\right)$. Observe that $v_{x \lambda+\delta t-\rho}^{\prime \prime}$ and $v_{x s \lambda+\delta t-\rho}^{\prime \prime}$ are mutually orthogonal with respect to $\langle\cdot, \cdot\rangle_{D}^{\prime \prime}:=T_{D_{\alpha}}^{D}\langle\cdot, \cdot\rangle_{x\left(\lambda-\nu_{\alpha}\right)+\delta t}^{\prime}$.

Recall $c_{x}^{\prime}=\left\langle v_{x\left(\lambda-\nu_{\alpha}\right)+\delta t-\rho}^{\prime}, v_{x\left(\lambda-\nu_{\alpha}\right)+\delta t-\rho}^{\prime}\right\rangle_{x\left(\lambda-\nu_{\alpha}\right)+\delta t}^{\prime}$. Let $\lambda_{\alpha}^{+} \in \Lambda$ be the highest weight of $L\left(\lambda_{\alpha}^{+}\right)_{A}=F\left(\nu_{\alpha}\right)_{A}$. From Theorem 4.3.3 we have

$$
\begin{aligned}
\left\langle v_{x \lambda+\delta t-\rho}^{\prime \prime}, v_{x \lambda+\delta t-\rho}^{\prime \prime}\right\rangle_{D}^{\prime \prime} & =(-1)^{\varepsilon\left(\lambda_{\alpha}^{+}-x \nu_{\alpha}\right)} D_{F\left(\nu_{\alpha}\right)_{A}}\left(x \nu_{\alpha}\right) a_{x \nu_{\alpha}}^{\prime \prime} c_{x}^{\prime} \quad \text { and } \\
\left\langle v_{x s \lambda+\delta t-\rho}^{\prime \prime}, v_{x s \lambda+\delta t-\rho}^{\prime \prime}\right\rangle_{D}^{\prime \prime} & =(-1)^{\varepsilon\left(\lambda_{\alpha}^{+}-x s \nu_{\alpha}\right)} D_{F\left(\nu_{\alpha}\right)_{A}}\left(x s \nu_{\alpha}\right) a_{x s \nu_{\alpha}}^{\prime \prime} c_{x}^{\prime}
\end{aligned}
$$

where

$$
\begin{aligned}
& a_{x \nu_{\alpha}}^{\prime \prime}=\prod_{\beta \in \Delta^{+} r>0, r+\left\langle x \nu_{\alpha}, \beta^{\vee}\right\rangle \geq 0}\left(\frac{\left(x\left(\lambda-\nu_{\alpha}\right)+\delta t, \beta^{\vee}\right)-r}{\left(x \lambda+\delta t, \beta^{\vee}\right)+r}\right)^{n\left(x \nu_{\alpha}+r \beta\right)} \text { and } \\
& a_{x s \nu_{\alpha}}^{\prime \prime}=\prod_{\beta \in \Delta^{+} r>0, r+\left\langle x s \nu_{\alpha}, \beta^{\vee}\right\rangle \geq 0}\left(\frac{\left(x s\left(\lambda-\nu_{\alpha}\right)+\delta t, \beta^{\vee}\right)-r}{\left(x s \lambda+\delta t, \beta^{\vee}\right)+r}\right)^{n\left(x s \nu_{\alpha}+r \beta\right)} .
\end{aligned}
$$

We compute which factors are zero at $t=0$.

Denominator of $a_{x \nu_{\alpha}}^{\prime \prime}$ : We require $r$ and $\beta>0$ such that $\left(x \lambda, \beta^{\vee}\right)=-r<0$. Then $s_{\beta} x \lambda-x \lambda=r \beta$ so $s_{\beta} x \lambda-x\left(\lambda-\nu_{\alpha}\right)=x \nu_{\alpha}+r \beta$. From 4.5.5 and from part ii) of Theorem 4.5 .1 we see that

$$
n\left(x \nu_{\alpha}+r \beta\right)= \begin{cases}1 & \text { if } s_{\beta} x=x s_{\alpha} \Rightarrow \beta=x \alpha \\ 0 & \text { otherwise. }\end{cases}
$$

We conclude that the denominator has exactly one factor, $\left(\delta t, x \alpha^{\vee}\right)$, which is zero at $t=0$.

Numerator of $a_{x \nu_{\alpha}}^{\prime \prime}$ : Suppose we have $\beta>0$ and $\left(x\left(\lambda-\nu_{\alpha}\right), \beta^{\vee}\right)=r>0$. Then $x\left(\lambda-\nu_{\alpha}\right)-s_{\beta} x\left(\lambda-\nu_{\alpha}\right)=r \beta$ so $s_{\beta} x \nu_{\alpha}-r \beta=s_{\beta} x \lambda-x\left(\lambda-\nu_{\alpha}\right)$. By 4.5.5 and by part ii) of Theorem 4.5 .1

$$
n\left(x \nu_{\alpha}+r \beta\right)=n\left(s_{\beta} x \nu_{\alpha}-r \beta\right)=\left\{\begin{array}{ll}
1 & \text { if } s_{\beta} x=x s_{\alpha} \\
0 & \text { otherwise. }
\end{array} \Rightarrow \beta=x \alpha\right.
$$

However, $\left(x\left(\lambda-\nu_{\alpha}\right), x \alpha^{\vee}\right)=0 \neq r$ and we deduce that the numerator has no factors which are zero at $t=0$.

Similarly, none of the factors in the numerator and the denominator of $a_{x s \nu_{\alpha}}^{\prime \prime}$ are zero at $t=0$.

Remark 4.5.4. The results of this section hold with any $z<z s$ in place of $x$. We define $v_{z \lambda+\delta t-\rho}^{\prime \prime}, v_{z s \lambda+\delta t-\rho}^{\prime \prime}, a_{z \nu_{\alpha}}^{\prime \prime}$, and $a_{z s \nu_{\alpha}}^{\prime \prime}$ analogously for all such $z \in W_{\lambda}$. 
4.6. A recursive formula in the difficult case. Here, we combine the results of the preceding subsections to deduce a recursive formula for computing signed Kazhdan-Lusztig polynomials for case b).

We will need Gabber and Joseph's description of $\bar{Y}_{j}$ where the form on $Y$ arises from the form on $Z$ and coherent continuation (cf. 4.4, 4.5, 4.6 of GJ81). Recall the exact sequence from Theorem 4.5.1 If we define $\bar{Y}_{j}^{x}=\left(\bar{Y}^{j} \cap \bar{X}\right) /\left(\bar{Y}^{j+1} \cap \bar{X}\right)$ and $\bar{Y}_{j}^{z}=\pi\left(\bar{Y}^{j}\right) / \pi\left(\bar{Y}^{j+1}\right)$ then there is a short exact sequence

$$
0 \rightarrow \bar{Y}_{j}^{x} \rightarrow \bar{Y}_{j} \rightarrow \bar{Y}_{j}^{z} \rightarrow 0 \text {. }
$$

For $M \in \mathrm{Ob} K_{C}$, we define $M^{+}$(resp. $M^{-}$) to be the smallest (resp. largest) submodule of $M$ for which $\theta_{\alpha}\left(M / M^{+}\right)=0$ (resp. $\left.\theta_{\alpha} M^{-}=0\right)$. We have the short exact sequences

$$
0 \rightarrow \bar{X}_{j+1}^{+} \rightarrow \bar{Y}_{j}^{x} \rightarrow \bar{X}_{j}^{-} \rightarrow 0
$$

and

$$
0 \rightarrow \bar{Z}_{j+1}^{-} \rightarrow \bar{Y}_{j}^{z} \rightarrow \bar{Z}_{j}^{+} \rightarrow 0
$$

(cf. 4.5 (2), 4.5 (4), Lemma 4.6 ii) and Proposition 4.7 of [GJ81]). This gives us the four-step filtration of $\bar{Y}_{j}$ :

\begin{tabular}{|c|c|c|c|}
\hline \multicolumn{4}{|c|}{$\bar{Y}_{j}$} \\
\hline \multicolumn{2}{|c|}{$\bar{Y}_{j}^{x}$} & \multicolumn{2}{c|}{$\bar{Y}_{j}^{z}$} \\
\hline $\bar{X}_{j+1}^{+}$ & $\bar{X}_{j}^{-}$ & $\bar{Z}_{j+1}^{-}$ & $\bar{Z}_{j}^{+}$ \\
\hline
\end{tabular}

Here we remark that in the paper GJ81, because 3.14 still holds and because we may modify Lemma 3.15 for invariant Hermitian forms, Hermitian analogues of results in sections 4.4 to 4.7 hold. We have:

Lemma 4.6.1. (cf. GJ81, Lemma 4.5.)

i) $\left\langle\bar{X}_{j+1}^{+}, \bar{Y}_{j}^{x}\right\rangle=0$

ii) $\left\langle\bar{X}_{j+1}^{+}, \operatorname{ker}\left(\bar{Y}_{j} \rightarrow \bar{Z}_{j}^{+}\right)\right\rangle=0$.

Furthermore, $\delta^{h}\left(\bar{X}_{j+1}^{+}\right) \cong \bar{Z}_{j}^{+}$. It follows that:

Proposition 4.6.2. Consider the four-step filtration of $\bar{Y}_{j} . \bar{X}_{j+1}^{+}$is paired with $\bar{Z}_{j}^{+}$, whence the signature character of $\bar{Y}_{j}$ is given by the signature characters of $\bar{X}_{j}^{-}$and $\bar{Z}_{j+1}^{-}$.

We will clarify the latter half of this statement, which is vague. First, we discuss the structure of $\bar{X}_{j}^{-}$and $\bar{Z}_{j+1}^{-}$, which is given (along with the structure of $\bar{X}_{i}^{ \pm}$and $\bar{Z}_{i}^{ \pm}$for any $i$ ) by Kazhdan-Lusztig polynomials and the following proposition:

Proposition 4.6.3. (GJ81, Lemma 3.6, 3.11.)

i) $\theta_{\alpha} L(z \lambda)=0$ if $z>z s$ and $\theta_{\alpha} L(z \lambda) \neq 0$ otherwise.

ii) When $z<z s, \theta_{\alpha} L(z \lambda)$ has a unique simple quotient and it is isomorphic to $L(z \lambda)$. The corresponding unique maximal submodule has unique simple submodule $L(z \lambda)$.

Recalling that $\bar{X}_{i}$ is semisimple, $\bar{X}_{i}^{+}$is a sum of simple submodules $L(z \lambda)$ for which $z>z s$ and $\bar{X}_{i}^{-}$is a sum of simple submodules $L(z \lambda)$ for which $z<z s$. Likewise for $\bar{Z}_{i}$. 
We study $\theta_{\alpha} L(z \lambda)$ when $z<z s$ in more detail. The functor $T_{\bar{D}_{\alpha}}^{\bar{D}}$ is a left and a right adjoint to $T_{\bar{D}}^{\bar{D}_{\alpha}}$ (cf. 3.4 of [GJ81] and (3.5) of Vog79a). It follows that

$$
\begin{aligned}
\operatorname{Hom}_{\mathfrak{g}}\left(L(z \lambda), T_{\bar{D}_{\alpha}}^{\overline{\bar{D}}} T_{\bar{D}}^{\bar{D}_{\alpha}} L(z \lambda)\right) & \cong \operatorname{Hom}_{\mathfrak{g}}\left(T_{\bar{D}}^{\bar{D}_{\alpha}} L(z \lambda), T_{\bar{D}}^{\bar{D}_{\alpha}} L(z \lambda)\right) \\
& \cong \operatorname{Hom}_{\mathfrak{g}}\left(T_{\bar{D}_{\alpha}}^{\bar{D}} T_{\bar{D}}^{\bar{D}_{\alpha}} L(z \lambda), L(z \lambda)\right)
\end{aligned}
$$

from which we obtain a chain complex

$$
0 \rightarrow L(z \lambda) \stackrel{i}{\rightarrow} \theta_{\alpha} L(z \lambda) \stackrel{p}{\rightarrow} L(z \lambda) \rightarrow 0
$$

(cf. Vog79a, Theorem 3.7). Because $L(z \lambda)$ is simple, the first map is injective and the second map is surjective.

Since $\theta_{\alpha} L(z \lambda)$ admits a non-degenerate invariant Hermitian form (for example, the form acquired through coherent continuation and the form on $L(z \lambda)$ ), $\delta^{h}\left(\theta_{\alpha} L(z \lambda)\right) \cong \theta_{\alpha} L(z \lambda)$. $\delta^{h}$ takes submodules of a module $M$ to quotients of $\delta^{h} M$ and quotients of $M$ to submodules of $\delta^{h} M$. Since $\delta^{h}$ does not take the submodule $L(z \lambda)$ of $\theta_{\alpha} L(z \lambda)$ to the submodule $L(z \lambda)$ of $\delta^{h}\left(\theta_{\alpha} L(z \lambda)\right)$, it follows that that submodule cannot be paired with itself, and hence it is paired with the quotient $L(z \lambda)$. We conclude:

Lemma 4.6.4. Suppose $z<z s$. Then $\operatorname{ch}_{s} \theta_{\alpha} L(z \lambda)=\operatorname{ch}_{s} U_{\alpha} L(z \lambda)$ where $U_{\alpha} L(z \lambda)$ is defined to be the cohomology of the complex

$$
0 \rightarrow L(z \lambda) \hookrightarrow \theta_{\alpha} L(z \lambda) \rightarrow L(z \lambda) \rightarrow 0 .
$$

$U_{\alpha}$ may be extended to semisimple modules via $U_{\alpha}(M \oplus N)=U_{\alpha} M \oplus U_{\alpha} N$. In particular, we may apply $U_{\alpha}$ to $\bar{Z}_{j}^{+}$. Since $\theta_{\alpha} M^{j}=\left(\theta_{\alpha} M\right)^{j}$ for $M \in \mathrm{Ob} K_{C}$ (cf. GJ81, Lemma 4.3 ii)), we see that $\bar{Y}_{j}=\theta_{\alpha} \bar{Z}_{j}=\theta_{\alpha} \bar{Z}_{j}^{+}$.

Proposition 4.6.5. (cf. GJ81, Proposition 4.7 iv).) There is a short exact sequence

$$
0 \rightarrow \bar{X}_{j}^{-} \rightarrow U_{\alpha} \bar{Z}_{j}^{+} \rightarrow \bar{Z}_{j+1}^{-} \rightarrow 0 .
$$

Furthermore, choosing the form $\theta_{\alpha}\langle\cdot, \cdot\rangle_{x \lambda+\delta t}$ on $Y$ :

$$
c h_{s} \bar{Y}_{j}=c h_{s} U_{\alpha} \bar{Z}_{j}^{+}=\operatorname{sgn}\left(\bar{c}_{x s}^{\prime \prime} \bar{c}_{x}^{\prime}\right) c h_{s} \bar{X}_{j}^{-}+\operatorname{sgn}\left(\bar{c}_{x}^{\prime \prime}\left(\delta, x \alpha^{\vee}\right) \bar{c}_{x}^{\prime}\right) c h_{s} \bar{Z}_{j}^{-}
$$

where for $z<z s$

$$
\begin{aligned}
c_{z}^{\prime \prime} & :=(-1)^{\varepsilon\left(\lambda_{\alpha}^{+}-z \nu_{\alpha}\right)} D_{F\left(\nu_{\alpha}\right)}\left(z \nu_{\alpha}\right) a_{z \nu_{\alpha}}^{\prime \prime}\left(\delta t, z \alpha^{\vee}\right) \\
\text { and } \quad c_{z s}^{\prime \prime} & :=(-1)^{\varepsilon\left(\lambda_{\alpha}^{+}-z s \nu_{\alpha}\right)} D_{F\left(\nu_{\alpha}\right)}\left(z s \nu_{\alpha}\right) a_{z s \nu_{\alpha}}^{\prime \prime} .
\end{aligned}
$$

Proof. This follows from our previous discussion, Theorems 4.3 .2 and 4.3 .3 and our analysis of $a_{z \nu_{\alpha}}^{\prime \prime}$ and $a_{z s \nu_{\alpha}}^{\prime \prime}$.

We discuss the signature character of an invariant Hermitian form on some $U_{\alpha} L(z \lambda)$. The process of coherent continuation in category $\mathcal{O}$ uniquely determines an invariant Hermitian form on $U_{\alpha} L(z \lambda)$ from a form on $L(z \lambda)$. Since $U_{\alpha} L(z \lambda)$ is semisimple by Vogan's Conjecture, it may have many other non-degenerate invariant Hermitian forms. For example, another natural form on $U_{\alpha} L(x \lambda)$ is the form on $\bar{Y}_{0}$ which arises from the Jantzen filtration of $\theta_{\alpha} Z=\theta_{\alpha} M(x \lambda+\delta t)$. The signature depends on $\delta$ while the form coming from coherent continuation in category $\mathcal{O}$ does not, and so the signatures may be different. The form given by the Jantzen 
filtration is the form in which we are interested. In the following computations, we will always study the form arising from the Jantzen filtration.

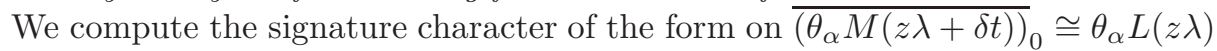
for $z<z s$. By Proposition 4.6.5 there is a short exact sequence

$$
0 \rightarrow{\overline{M(z s \lambda+\delta t)_{0}}}^{-} \rightarrow U_{\alpha}{\overline{M(z \lambda+\delta t})_{0}} \rightarrow \overline{M(z \lambda+\delta t)}_{1}^{-} \rightarrow 0 .
$$

By $c h_{s} U_{\alpha} \overline{M(z \lambda+\delta t)}$, we mean the signature character of the form given by the Jantzen filtration in the direction $\delta$. By our analysis of the denominators and numerators of $a_{z \nu_{\alpha}}^{\prime \prime}$ and of $a_{z s \nu_{\alpha}}^{\prime \prime}$, the denominators of $c_{z}^{\prime \prime}$ and $c_{z s}^{\prime \prime}$ do not vanish at $t=0$. From Jantzen's determinant formula and our short exact sequence above,

$$
\begin{aligned}
c h_{s} U_{\alpha} \overline{M(z \lambda+\delta t)_{0}}= & \operatorname{sgn}\left(\bar{c}_{z s}^{\prime \prime} \bar{c}_{z}^{\prime}\right) c h_{s} L(z s \lambda) \\
& +\operatorname{sgn}\left(\bar{c}_{z}^{\prime \prime}\left(\delta, z \alpha^{\vee}\right) \bar{c}_{z}^{\prime}\right) \sum_{y \in W_{\lambda} \mid y>y s} a_{y, 1}^{z \lambda, w} c h_{s} L(y \lambda) .
\end{aligned}
$$

Using this in conjunction with the previous proposition gives:

Proposition 4.6.6. If $x, y \in W_{\lambda}$ are such that $x<x s$ and $y>y s$ and $x>y$ then:

$$
\begin{aligned}
\operatorname{sgn}\left(\bar{c}_{x s}^{\prime \prime} \bar{c}_{x}^{\prime}\right) P_{w_{\lambda} x s, w_{\lambda} y}^{\lambda, w}(q) & +\operatorname{sgn}\left(\bar{c}_{x}^{\prime \prime}\left(\delta, x \alpha^{\vee}\right) \bar{c}_{x}^{\prime}\right) q P_{w_{\lambda} x, w_{\lambda} y}^{\lambda, w}(q) \\
= & \sum_{z \in W_{\lambda} \mid z<z s} \operatorname{sgn}\left(\bar{c}_{z}^{\prime \prime}\left(\delta, z \alpha^{\vee}\right) \bar{c}_{z}^{\prime}\right) a_{y, 1}^{z \lambda, w} q^{\frac{\ell(z)-\ell(y)+1}{2}} P_{w_{\lambda} x, w_{\lambda} z}^{\lambda, w}(q) \\
& +\operatorname{sgn}\left(\bar{c}_{y s}^{\prime \prime}\left(\delta, y s \alpha^{\vee}\right) \bar{c}_{y s}^{\prime}\right) P_{w_{\lambda} x, w_{\lambda} y s}^{\lambda, w}(q) .
\end{aligned}
$$

We discuss the values of $\operatorname{sgn}\left(\bar{c}_{z}^{\prime \prime}\right), \operatorname{sgn}\left(\bar{c}_{z s}\right)$ and $\operatorname{sgn}\left(\bar{c}_{z}^{\prime}\right)$ for $z<z s$.

Lemma 4.6.7. For an integral weight $\nu$ and for all $w \in W$,

$$
\operatorname{sgn}\left(D_{F(\nu)}(w \nu)\right)=1
$$

(see Theorem 4.3 .3 for notation).

Proof. We prove this by induction on $\ell(w)$. Clearly this is true for $w=1$. We may assume $\nu$ to be dominant and let $v_{\nu}$ be the canonical generator of $F(\nu)$. Suppose the lemma holds for $w \in W$ and $s_{\alpha}$ is a simple reflection such that $s_{\alpha} w>w$. Let $a \in U\left(\mathfrak{n}^{o p}\right)$ be such that $a v_{\nu}$ is a vector of weight $w \nu$ in $F(\nu)$. Now $s_{\alpha} w>w$, so $\left(w \nu, \alpha^{\vee}\right)>0$. Let $n_{\alpha}=\left(w \nu, \alpha^{\vee}\right)=\left(\nu, w^{-1} \alpha^{\vee}\right) \in \mathbb{Z}^{\geq 0} . s_{\alpha} w \nu=w \nu-n_{\alpha} \alpha$ and so $Y_{\alpha}^{n_{\alpha}} a v_{\nu}$ is a vector of weight $s_{\alpha} w \nu$ in $F(\nu)$. Because $a v_{\nu}$ is a vector of extremal weight $w \nu$ and because the set of weights of $F(\nu)$ is convex, $Y_{\alpha}^{n_{\alpha}} a v_{\nu} \in F(\nu)$ implies $X_{\alpha} a v_{\nu}=0$. Therefore

$$
\begin{aligned}
\left(Y_{\alpha}^{n_{\alpha}} a v_{\nu}, Y_{\alpha}^{n_{\alpha}} a v_{\nu}\right) & =\left(\sigma\left(Y_{\alpha}^{n_{\alpha}} a\right) Y_{\alpha}^{n_{\alpha}} a v_{\nu}, v_{\nu}\right)=\left(\sigma(a) X_{\alpha}^{n_{\alpha}} Y_{\alpha}^{n_{\alpha}} a v_{\nu}, v_{\nu}\right) \\
\left(\text { from } X_{\alpha} a v_{\nu}=0\right) & =\left(\sigma(a) p\left(X_{\alpha}^{n_{\alpha}} Y_{\alpha}^{n_{\alpha}}\right) a v_{\nu}, v_{\nu}\right) \\
\left(\text { from } \mathfrak{s l}_{2} \text { theory }\right) & =w \nu\left(H_{\alpha}\left(H_{\alpha}-1\right) \cdots\left(H_{\alpha}-\left(n_{\alpha}-1\right)\right)\right) \cdot\left(\sigma(a) a v_{\nu}, v_{\nu}\right) .
\end{aligned}
$$

Now $w \nu\left(H_{\alpha}\left(H_{\alpha}-1\right) \cdots\left(H_{\alpha}-\left(n_{\alpha}-1\right)\right)\right)>0$ since $w \nu\left(H_{\alpha}\right)=n_{\alpha}$. By our induction hypothesis, $\left(\sigma(a) a v_{\nu}, v_{\nu}\right)>0$. Thus $\left(Y_{\alpha}^{n_{\alpha}} a v_{\nu}, Y_{\alpha}^{n_{\alpha}} a v_{\nu}\right)>0$, proving our lemma.

Remark 4.6.8. We may also prove the lemma using the following unpublished result of Birgit Speh: if $\mathfrak{h}$ is a compact Cartan subalgebra, then given the finite dimensional representation of highest weight $\lambda_{0}$, the Shapovalov form is definite on each weight space, with the form being positive definite (resp. negative definite) 
on the $\lambda_{0}-\mu$ weight space if $\varepsilon(\mu)=0$ (resp. $\varepsilon(\mu)=1$ ). Again, we may take $\nu$ to be dominant. Comparing what Speh's formula and Lemma 4.3.4 imply for the signature of the one-dimensional weight space corresponding to $w \nu$, we have $(-1)^{\varepsilon(\nu-w \nu)}=\operatorname{sgn}\left(D_{F(\nu)}(w \nu)\right)(-1)^{\varepsilon(\nu-w \nu)}$ from which the lemma follows.

Lemma 4.6.9. For $z<z s \in W_{\lambda}$ :
i) $\operatorname{sgn}\left(\bar{a}_{-z \nu_{\alpha}}^{\prime}\right)=1$.
ii) $\operatorname{sgn}\left(\overline{a_{z \nu_{\alpha}}^{\prime \prime}\left(\delta t, z \alpha^{\vee}\right)}\right)=-1$.
iii) $\operatorname{sgn} \bar{a}_{z s \nu_{\alpha}}^{\prime \prime}=1$.

Proof. i): Consider (4.5.3). If $\left(-z \nu_{\alpha}, \beta^{\vee}\right) \geq 0$ then $n\left(-z \nu_{\alpha}+r \beta\right)=0$ for $r>0$. If $\left(-z \nu_{\alpha}, \beta^{\vee}\right)<0$, then the index for the second product starts at $r=-\left(-z \nu_{\alpha}, \beta^{\vee}\right)$.

$$
-z \nu_{\alpha}-\left(-z \nu_{\alpha}, \beta^{\vee}\right) \beta=s_{\beta}\left(-z \nu_{\alpha}\right)
$$

is an extremal weight of $F\left(-\nu_{\alpha}\right)$. Therefore $n\left(-z \nu_{\alpha}+r \beta\right)=0$ for $r>-\left(-z \nu_{\alpha}, \beta^{\vee}\right)$. Therefore $\bar{a}_{-z \nu_{\alpha}}^{\prime}$ may be written

$$
\begin{aligned}
\bar{a}_{-z \nu_{\alpha}}^{\prime} & =\prod_{\beta \in \Delta^{+}(\mathfrak{g}, \mathfrak{h}),\left(-z \nu_{\alpha}, \beta^{\vee}\right)<0}\left(\frac{\left(z \lambda, \beta^{\vee}\right)+\left(-z \nu_{\alpha}, \beta^{\vee}\right)}{\left(z \lambda-z \nu_{\alpha}, \beta^{\vee}\right)-\left(-z \nu_{\alpha}, \beta^{\vee}\right)}\right)^{1} \\
& =\prod_{\beta \in \Delta^{+}(\mathfrak{g}, \mathfrak{h}),\left(-z \nu_{\alpha}, \beta^{\vee}\right)<0} \frac{\left(z\left(\lambda-\nu_{\alpha}\right), \beta^{\vee}\right)}{\left(z \lambda, \beta^{\vee}\right)} .
\end{aligned}
$$

$\lambda-\nu_{\alpha}$ lies in the closure of the antidominant Weyl chamber, which is the Weyl chamber to which $\lambda$ belongs. Since $\left(\lambda-\nu_{\alpha}, \beta^{\vee}\right) \neq 0$ for $\beta \neq \alpha$, we conclude that $\operatorname{sgn}\left(z\left(\lambda-\nu_{\alpha}\right), \beta^{\vee}\right)=\operatorname{sgn}\left(z \lambda, \beta^{\vee}\right)$ for $\beta \neq z \alpha$. Observing that $\left(-z \nu_{\alpha}, z \alpha^{\vee}\right)>0$, we conclude that $\operatorname{sgn}\left(\bar{a}_{-z \nu_{\alpha}}^{\prime}\right)=1$.

ii): As in the previous case,

$$
\overline{a_{z \nu_{\alpha}}^{\prime \prime}\left(\delta t, z \alpha^{\vee}\right)}=\left(z \lambda, z \alpha^{\vee}\right) \prod_{\beta \in \Delta^{+}(\mathfrak{g}, \mathfrak{h}) \backslash\{z \alpha\},\left(z \nu_{\alpha}, \beta^{\vee}\right)<0}\left(\frac{\left(z \lambda, \beta^{\vee}\right)}{\left(z\left(\lambda-\nu_{\alpha}\right), \beta^{\vee}\right)}\right)^{1}
$$

$\operatorname{so~} \operatorname{sgn}\left(\overline{a_{z \nu_{\alpha}}^{\prime \prime}\left(\delta t, z \alpha^{\vee}\right)}\right)=-1$

iii): As in the first case,

$$
\bar{a}_{z s \nu_{\alpha}}^{\prime \prime}=\prod_{\beta \in \Delta^{+}(\mathfrak{g}, \mathfrak{h}),\left(z s \nu_{\alpha}, \beta^{\vee}\right)<0} \frac{\left(z s \lambda, \beta^{\vee}\right)}{\left(z s\left(\lambda-\nu_{\alpha}\right), \beta^{\vee}\right)} .
$$

Since $\left(z s \nu_{\alpha}, z \alpha^{\vee}\right)>0$, we conclude that $\operatorname{sgn}\left(\bar{a}_{z s \nu_{\alpha}}^{\prime \prime}\right)=1$.

Combining the results of this subsection, cancelling out common factors of $(-1)^{\varepsilon\left(\lambda_{\alpha}^{+}+\lambda_{\alpha}^{-}\right)}$, and observing that $x \nu_{\alpha}-x s \nu_{\alpha}=x\left(\left(\nu_{\alpha}, \alpha^{\vee}\right) \alpha\right)=x\left(\left(\lambda, \alpha^{\vee}\right) \alpha\right)$, we arrive at:

Theorem 4.6.10. Letting $s=s_{\alpha}$ be a simple reflection, the signed Kazhdan-Lusztig polynomials are defined by the intial conditions $P_{x, x}^{\lambda, w}=1, P_{x, y}^{\lambda, w}=0$ for $x>y$ and the recursive formulas:

a) $P_{w_{\lambda} x, w_{\lambda} y}^{\lambda, w}=\operatorname{sgn}(-w \rho, x \alpha) \varepsilon\left(H_{x \alpha,-\left(\lambda, \alpha^{\vee}\right)}, x s\right) P_{w_{\lambda} x s, w_{\lambda} y}^{\lambda, w}$ if $y s>y$ and $x s>$ $x \geq y$

$\left.\mathrm{a}^{\prime}\right) P_{w_{\lambda} x, w_{\lambda} y}^{\overline{\lambda, w}}=\operatorname{sgn}(-w \rho, \alpha) \varepsilon\left(H_{\alpha,\left(s x \lambda, \alpha^{\vee}\right)}, s x\right) P_{w_{\lambda} s x, w_{\lambda} y}^{\lambda, w}$ if $s y>y$ and $s x>$ $x \geq y$ 
b) If $x, y \in W_{\lambda}$ are such that $x<x s$ and $y>y s$ and $x>y$ then:

$$
\begin{aligned}
& -(-1)^{\varepsilon\left(\left(\lambda, \alpha^{\vee}\right) x \alpha\right)} P_{w_{\lambda} \lambda, w s, w_{\lambda} y}^{\lambda, w}(q)+\operatorname{sgn}\left(\delta, x \alpha^{\vee}\right) q P_{w_{\lambda} x, w_{\lambda} y}^{\lambda, w}(q) \\
& =\sum_{z \in W_{\lambda} \mid z<z s} \operatorname{sgn}\left(\delta, z \alpha^{\vee}\right) a_{y, 1}^{z \lambda, w} q^{\frac{\ell(z)-\ell(y)+1}{2}} P_{w_{\lambda}^{\lambda} x, w_{\lambda} z}^{\lambda, w}(q)+\operatorname{sgn}\left(\delta, y s \alpha^{\vee}\right) P_{w_{\lambda} x, w_{\lambda} y s}^{\lambda, w}(q) .
\end{aligned}
$$

\section{Some Examples}

Example 1: $\mathfrak{g}_{0}=\mathfrak{s o}(2)$. We have $\mathfrak{h}=\mathfrak{t}$. Let $\Delta^{+}(\mathfrak{g}, \mathfrak{h})=\left\{\alpha_{1}\right\}$ and let $\lambda_{1}$ be the corresponding fundamental weight.

Irreducible Verma modules: Choose $\lambda \in \mathfrak{h}^{*}$ so that $\left(\lambda, \alpha_{1}^{\vee}\right) \in(n, n+1)$ where $n \in \mathbb{Z}_{\geq 0}$. Then $\lambda \in A\left(n \lambda_{1}, w_{0}\right)$. The reducibility hyperplanes separating the alcove $a A_{0}$ containing $\lambda$ and $\widetilde{a} A_{0}$ are $H_{\alpha_{1}, 1}, H_{\alpha_{1}, 2}, \ldots H_{\alpha_{1}, n}$. In the setup of Theorem 3.2.4 we choose the path so that $r_{1}=s_{\alpha_{1}, n}, r_{2}=s_{\alpha_{1}, n-1}, \ldots, r_{n}=s_{\alpha_{1}, 1}$. Suppose $S \subset\{1,2, \ldots, n\}$ and $|S| \geq 2$. Then $\overline{r_{i_{1}}} C_{i_{2}-1}$ and $\overline{r_{i_{1}}} C_{i_{2}}$ lie in the Wallach region, and thus $\varepsilon\left(\overline{r_{i_{1}}} C_{i_{2}-1}, \overline{r_{i_{1}}} C_{i_{2}}\right)=0$. Therefore $\varepsilon(S)=0$ for $|S| \geq 2$. For our choice of path, note that $C_{i} \supset(n-i, n-i+1)$, whence $\varepsilon(\{i\})=\varepsilon\left(C_{i-1}, C_{i}\right)=$ $\varepsilon\left(H_{\alpha_{1}, n-i+1}, s_{1}\right)=\delta_{\alpha_{1}}^{n-i+1}=1$ (see Definition 5.2.16 and Lemma 5.2.17 or Theorem 6.12 of Yee05]). Substituting these values into Theorem 3.2.4

$$
\begin{aligned}
R^{A\left(n \lambda_{1}, w_{0}\right)}=\operatorname{ch}_{s} M(\lambda) & =\frac{\sum_{i=1}^{n} 2 e^{\overline{r_{i}} r_{i} \lambda-\rho}+e^{\lambda-\rho}}{\prod_{\alpha \in \Delta^{+}(\mathfrak{p}, \mathfrak{t})}\left(1-e^{-\alpha}\right) \prod_{\alpha \in \Delta^{+}(\mathfrak{k}, \mathfrak{t})}\left(1-e^{-\alpha}\right)} \\
& =\frac{\sum_{i=1}^{n} 2 e^{\lambda-i \alpha_{1}-\rho}+e^{\lambda-\rho}}{1+e^{-\alpha_{1}}} \\
& =\frac{\sum_{i=1}^{n} e^{\lambda-(i-1) \alpha_{1}-\rho}+e^{\lambda-i \alpha_{1}-\rho}}{1+e^{-\alpha_{1}}} \\
& =e^{\lambda-\rho}+e^{\lambda-\rho-\alpha_{1}-\rho}+\cdots+e^{\lambda-(n-1) \alpha_{1}-\rho}+\frac{e^{\lambda-n \alpha_{1}-\rho}}{1+e^{-\alpha_{1}}} .
\end{aligned}
$$

Irreducible highest weight modules: Let $\lambda=-n \lambda_{1}$ for some $n \in \mathbb{Z}^{+}$. Since $\lambda$ is in the Wallach region, taking $n=0$ in the above formula:

$$
\operatorname{ch}_{s} L(\lambda)=\operatorname{ch}_{s} M(\lambda)=\frac{e^{\lambda-\rho}}{1+e^{-\alpha}} .
$$

According to Theorem 4.6 .10

$$
1=P_{w_{0}, w_{0}}^{\lambda, w_{0}}=\operatorname{sgn}\left(-w_{0} \rho, \alpha_{1}\right) \varepsilon\left(H_{\alpha_{1}, n}, s_{1}\right) P_{w_{0} s_{1}, w_{0}}^{\lambda, w}=\delta_{\alpha_{1}}^{n} P_{w_{0} s_{1}, w_{0}}^{\lambda, w}=P_{w_{0} s_{1}, w_{0}}^{\lambda, w_{0}}
$$

by Lemma 5.2.17 or Theorem 6.12 of Yee05. Substituting the values we have computed into Theorem 3.2 .3

$$
\begin{aligned}
\operatorname{ch}_{s} L\left(s_{1} \lambda\right) & =R^{A\left(s_{1} \lambda, w_{0}\right)}\left(s_{1} \lambda\right)-P_{w_{0} s_{1}, w_{0}}^{\lambda, R_{1}} R^{A\left(\lambda, w_{0}\right)}(\lambda) \\
& =R^{A\left(n \lambda_{1}, w_{0}\right)}\left(s_{1} \lambda\right)-R^{A\left(-n \lambda_{1}, w_{0}\right)}\left(s_{1} \lambda-n \alpha_{1}\right) \\
& =R^{A\left(n \lambda_{1}, w_{0}\right)}\left(s_{1} \lambda\right)-R^{A\left(0 \lambda_{1}, w_{0}\right)}\left(s_{1} \lambda-n \alpha_{1}\right) \\
& =\left(e^{s_{1} \lambda-\rho}+\cdots+e^{s_{1} \lambda-(n-1) \alpha_{1}-\rho}+\frac{e^{s_{1} \lambda-n \alpha_{1}-\rho}}{1+e^{-\alpha_{1}}}\right)-\left(\frac{e^{s_{1} \lambda-n \alpha_{1}-\rho}}{1+e^{-\alpha_{1}}}\right) \\
& =e^{s_{1} \lambda-\rho}+e^{s_{1} \lambda-\alpha_{1}-\rho}+\cdots+e^{s_{1} \lambda-(n-1) \alpha_{1}-\rho} .
\end{aligned}
$$




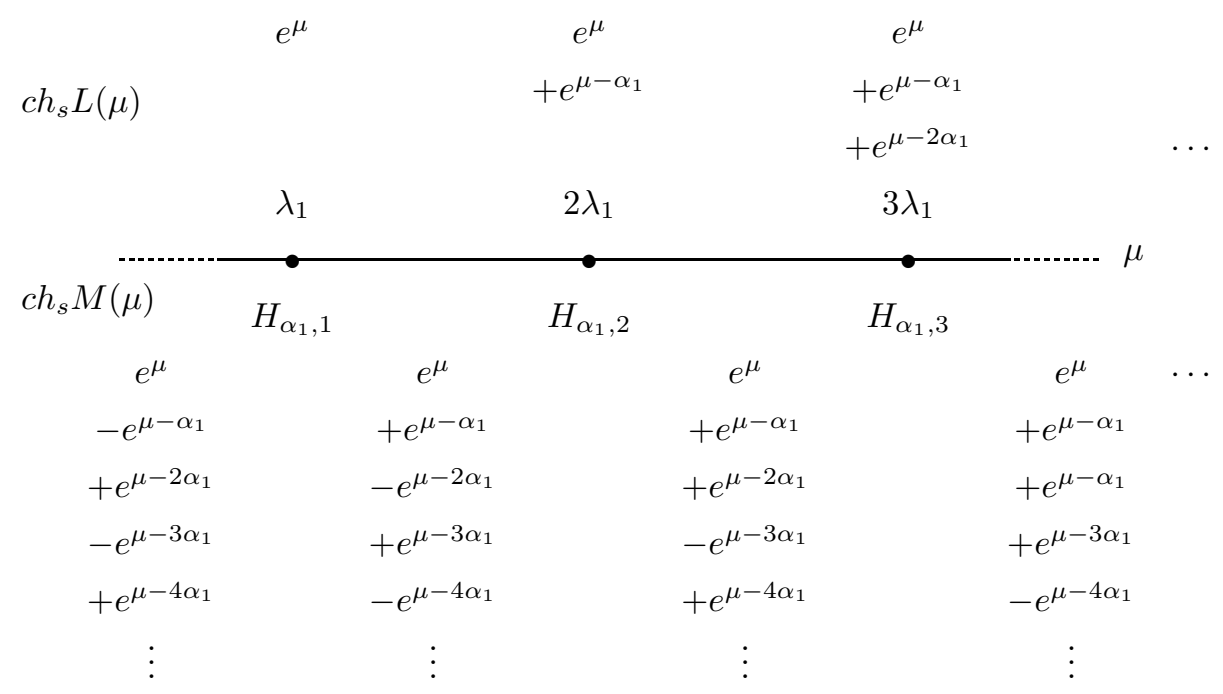

FIGURE 1. $\mathfrak{s u}(2)$

Example 2: $\mathfrak{g}_{0}=\mathfrak{s l}(2, \mathbb{R})$. We may proceed as in the previous example, but substitute $\delta_{\alpha_{1}}=-1$ instead of $\delta_{\alpha_{1}}=1$.

Irreducible Verma modules: For $\lambda \in \mathfrak{h}^{*}$ such that $\left(\lambda, \alpha_{1}^{\vee}\right) \in(n, n+1)$ where $n \in \mathbb{Z}_{\geq 0}$ :

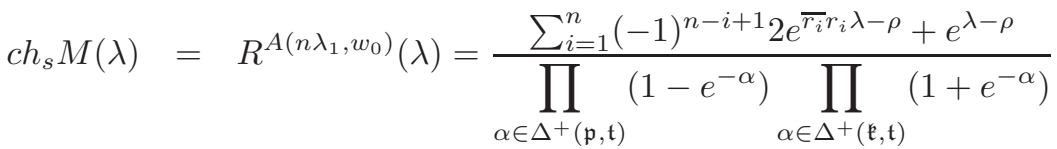

$$
\begin{aligned}
& =\frac{\sum_{i=1}^{n}(-1)^{i} 2 e^{\lambda-i \alpha_{1}-\rho}+e^{\lambda-\rho}}{1-e^{-\alpha_{1}}} \\
& =e^{\lambda-\rho}-e^{\lambda-\rho-\alpha_{1}-\rho}+\cdots+(-1)^{n-1} e^{\lambda-(n-1) \alpha_{1}-\rho}+(-1)^{n} \frac{e^{\lambda-n \alpha_{1}-\rho}}{1-e^{-\alpha_{1}}} .
\end{aligned}
$$

Irreducible highest weight modules: For $\lambda=-n \lambda_{1}$ where $n \in \mathbb{Z}^{+}$:

$$
\operatorname{ch} L(\lambda)=\operatorname{ch}_{s} M(\lambda)=\frac{e^{\lambda-\rho}}{1-e^{-\alpha_{1}}} .
$$

Since $P_{w_{0} s_{1}, s_{0}}^{\lambda, w_{0}}=(-1)^{n}$, we have

$$
\begin{aligned}
\operatorname{ch}_{s} L\left(s_{1} \lambda\right) & =R^{A\left(s_{1} \lambda, w_{0}\right)}\left(s_{1} \lambda\right)-P_{w_{0} s_{1}, w_{0}}^{\lambda, w_{0}} R^{A\left(\lambda, w_{0}\right)}(\lambda) \\
& =\left(\sum_{i=0}^{n-1}(-1)^{i} e^{s_{1} \lambda-i \alpha_{1}-\rho}+(-1)^{n} \frac{e^{s_{1} \lambda-n \alpha_{1}-\rho}}{1-e^{-\alpha_{1}}}\right)-(-1)^{n}\left(\frac{e^{s_{1} \lambda-n \alpha_{1}-\rho}}{1-e^{-\alpha_{1}}}\right) \\
& =e^{s_{1} \lambda-\rho}-e^{s_{1} \lambda-\alpha_{1}-\rho}+\cdots+(-1)^{n-1} e^{s_{1} \lambda-(n-1) \alpha_{1}-\rho} .
\end{aligned}
$$

\section{REFERENCES}

[Bar83] Dan Barbasch. Filtrations on Verma modules. Ann. Sci. École Norm. Sup. (4), 16(3):489-494, 1983 


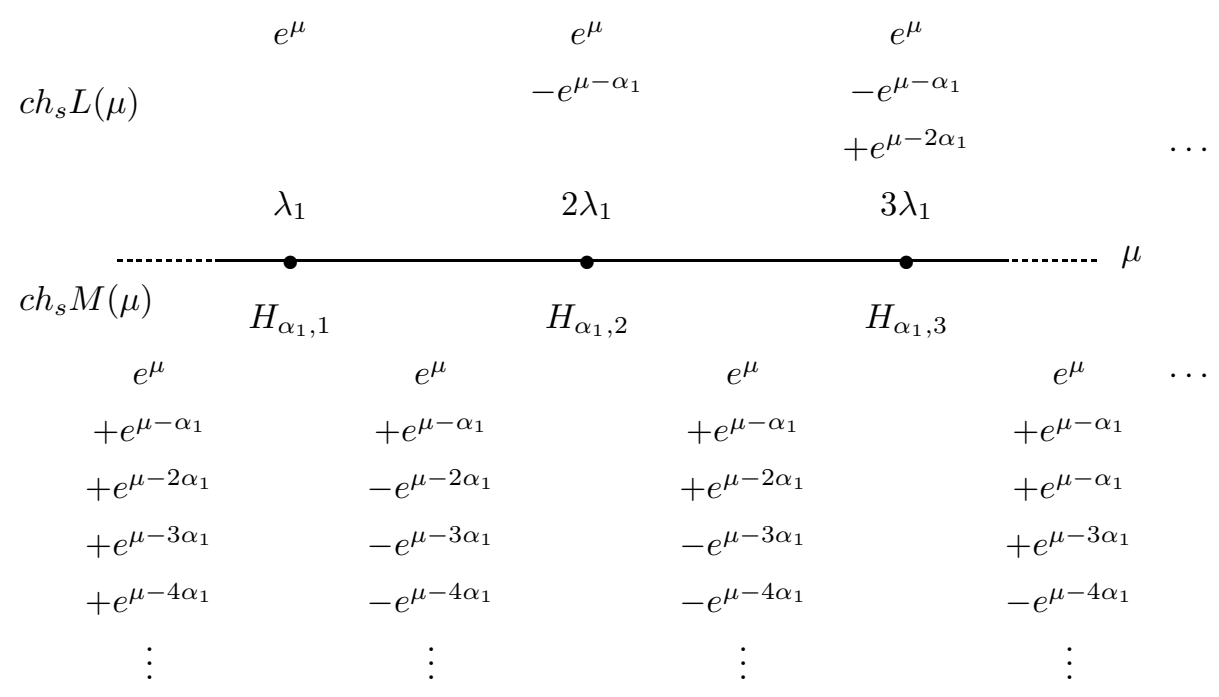

FigURE 2. $\mathfrak{s l}(2)$

[BB81] Alexandre Beullinson and Joseph Bernstein. Localisation de g-modules. C. R. Acad. Sci. Paris Sér. I Math., 292(1):15-18, 1981.

[BB93] A. Beilinson and J. Bernstein. A proof of Jantzen conjectures, volume 16 of Adv. Soviet Math. Amer. Math. Soc., Providence, RI, 1993.

[BGG71] I. N. Bernstein, I. M. Gelfand, and S. I. Gelfand. Structure of representations that are generated by vectors of higher weight. Funckcional. Anal. i Priložen., 5(1):1-9, 1971.

[BGG76] I. N. Bernstein, I. M. Gelfand, and S. I. Gelfand. A certain category of $\mathfrak{g}$-modules. Functional Anal. Appl., 10:87-92, 1976.

[BK81] J.-L. Brylinski and M. Kashiwara. Kazhdan-Lusztig conjecture and holonomic systems. Invent. Math., 64(3):387-410, 1981.

[Dix96] Jacques Dixmier. Enveloping Algebras. Number 11 in Graduate Studies in Mathematics. American Mathematical Society, Providence, RI, 1996.

[DL77] Vinay Deodhar and James Lepowsky. On multiplicity in the Jordan-Hölder series of Verma modules. J. Algebra, 49(2):512-524, 1977.

[EW80] Thomas Enright and Nolan R. Wallach. Notes on homological algebra and representations of Lie algebras. Duke Math. J., 47(1):1-15, 1980.

[GJ81] O. Gabber and A. Joseph. Towards the Kazhdan-Lusztig conjecture. Ann. Sci École Norm. Sup. (4), 14(3):261-302, 1981.

[Hum72] James E. Humphreys. Introduction to Lie Algebras and Representation Theory. Number 9 in Graduate Texts in Mathematics. Springer-Verlag, New York, 1972.

[Hum78] James E. Humphreys. Finite and infinite dimensional modules for semisimple Lie algebras. In Lie theories and their applications (Proc. Ann. Sem. Canad. Math. Congr., Queen's Univ., Kingston, Ont., 1977), volume 48 of Queen's Papers in Pure and Appl. Math., pages 1-64. Queen's Univ., Kingston, Ont., 1978.

[Hum90] James E. Humphreys. Reflection groups and Coxeter groups. Number 29 in Cambridge Studies in Advanced Mathematics. Cambridge University Press, Cambridge, 1990.

[Jan73] Jens Carsten Jantzen. Darstellungen halbeinfacher algebraischer Gruppen und zugeordnete kontravariante Formen. Bonn. Math. Schr., (67):v+124, 1973.

[Jan74] Jens Carsten Jantzen. Zur Charakterformel gewisser Darstellungen halbeinfacher Gruppen und Lie-Algebren. Math. Z., 140:127-149, 1974

[Jan79] Jens Carsten Jantzen. Moduln mit einem höchsten Gewicht. Number 750 in Lecture Notes in Mathematics. Springer, Berlin, 1979.

[KL79] D. Kazhdan and G. Lusztig. Representations of Coxeter groups and Hecke algebras. Invent. Math., 53:165-184, 1979. 
[Kna96] Anthony W. Knapp. Lie Groups Beyond an Introduction. Number 140 in Progress in Mathematics. Birkhäuser, Boston, 1996.

[Kos75] Bertram Kostant. On the tensor product of a finite and an infinite dimensional representation. J. Functional Analysis, 20(4):257-285, 1975.

[KV95] Anthony W. Knapp and David A. Vogan. Cohomological induction and unitary representations. Number 45 in Princeton Mathematical Series. Princeton University Press, Princeton, New Jersey, 1995.

[MFF86] F.G. Malikov, B.L. Feigin, and D.B. Fuks. Singular vectors in Verma modules over Kac-Moody algebras. Functional Analysis Applications, 20(2):103-113, 1986.

[SV80] Birgit Speh and David A. Vogan. Reducibility of generalized principal series representations. Acta Math., 145(3-4):227-299, 1980.

[Ver68] Daya-Nand Verma. Structure of certain induced representations of complex semisimple Lie algebras. Bull. Amer. Math. Soc., 74:160-166, 1968.

[Vog79a] David A. Vogan. Irreducible characters of semisimple Lie groups I. Duke Math. J., 46:61-108, 1979.

[Vog79b] David A. Vogan. Irreducible characters of semisimple Lie groups II. The KazhdanLusztig conjectures. Duke Math. J., 46:805-859, 1979.

[Vog81] David A. Vogan. Representations of real reductive Lie groups. Number 15 in Progress in Mathematics. Birkhäuser, Boston, Massachusetts, 1981.

[Vog84] David A. Vogan. Unitarizability of certain series of representations. Ann. of Math. (2), 120(1):141-187, 1984.

[Wal84] Nolan R. Wallach. On the unitarizability of derived functor modules. Invent. Math., 78(1):131-141, 1984.

[Yee05] Wai Ling Yee. The signature of the Shapovalov form on irreducible Verma modules. Representation Theory, 9:638-677, 2005.

Department of Mathematical and Statistical Sciences, University of Alberta, Edmonton, Alberta, CANADA

E-mail address: wlyee@math.ualberta.ca 